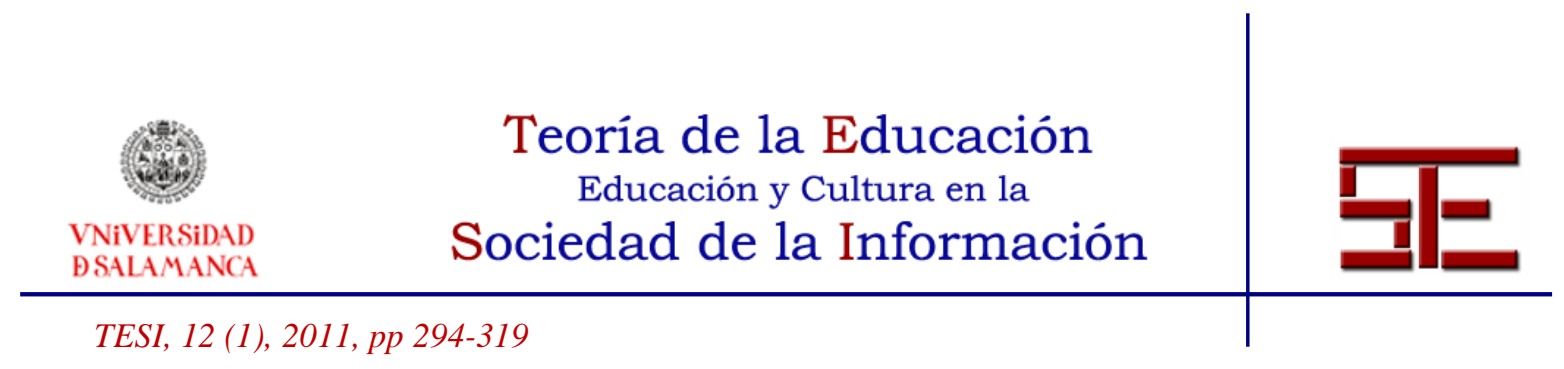

\title{
EL DESARROLLO DE COMPETENCIAS COMUNICATIVAS A TRAVÉS DE SEMINARIOS TRANSVERSALES ECTS: UNA EXPERIENCIA EN LA UNIVERSIDAD DE JAÉN
}

Resumen: El presente artículo describe una experiencia novedosa en el desarrollo de competencias genéricas y específicas y en el fomento de la interdisciplinariedad y la transversalidad. Se trata de un sistema de seminarios ECTS - que también entroncan con el Plan de Acción Tutorial - que se han desarrollado en la Licenciatura en Filología Inglesa de la Universidad de Jaén durante este curso académico (2008-2009). Con ellos, hemos pretendido fomentar la coordinación entre el profesorado de dicha titulación, superar el individualismo que suele caracterizar a las actuaciones docentes en educación terciaria y realizar la transición desde la cultura tradicional de la enseñanza memorística y transmisiva hacia la cultura del aprendizaje crítico y significativo basado en competencias y no meramente contenidos. Tras justificar la necesidad de una experiencia de este tipo y describir su desarrollo, contenidos y evaluación en detalle, se presenta el estudio descriptivo que la ha acompañado. Dicha investigación nos ha permitido concluir que el sistema de seminarios ECTS ha resultado altamente beneficioso para el alumnado participante. Éste ha sido consciente de la existencia, desarrollo y utilidad de una serie de competencias genéricas y específicas esenciales para el filólogo inglés. Consideramos que, en nuestro contexto, estos seminarios ECTS han constituido un importante primer paso en la integración, enseñanza y evaluación de competencias en el currículo.

Palabras clave: competencias; ECTS; EEES; transversalidad; competencia comunicativa.

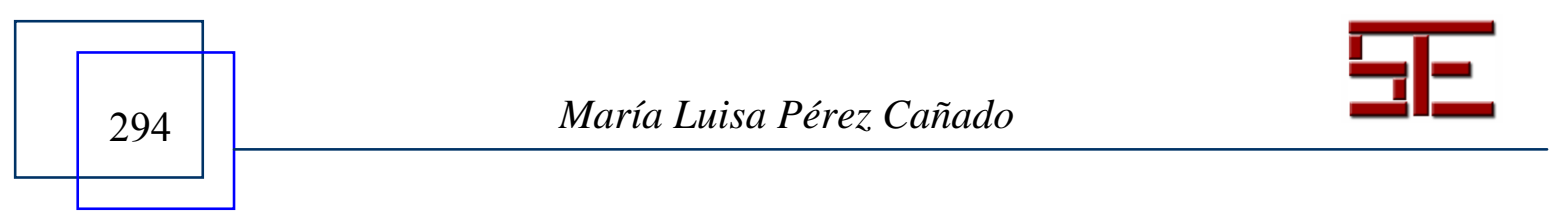




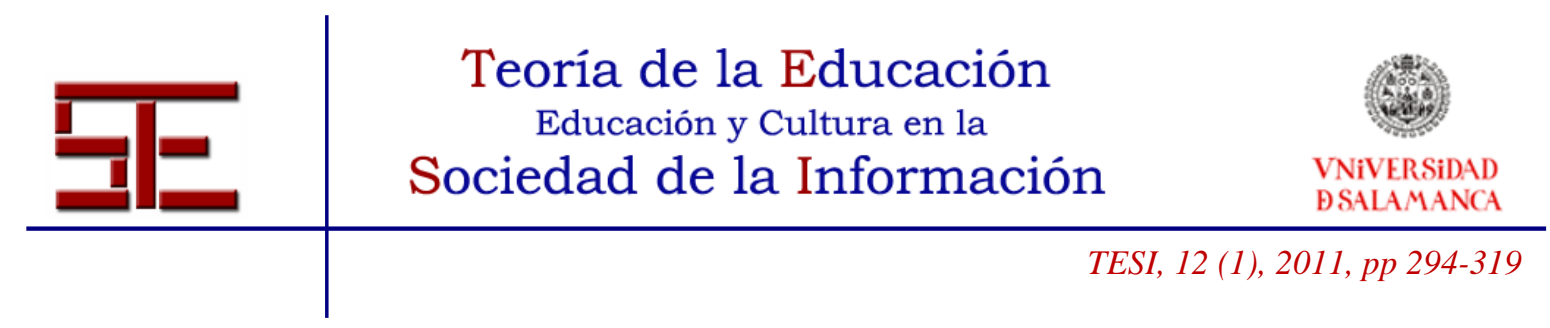

\title{
THE DEVELOPMENT OF COMMUNICATIVE COMPETENCES VIA CROSS- CURRICULAR ECTS SEMINARS
}

\begin{abstract}
This article describes an innovative experience in the development of crosscurricular generic and subject-specific competencies carried out via a system of ECTS seminars which has been developed within the degree in English Philology at the University of Jaén during the academic year 2008-2009. The aim of these seminars has been to reinforce coordination amongst professors, to overcome the oftentimes worrying individualism which characterizes teaching practices at tertiary level, and to make the transition from the transmission of information model based on ex-cathedra lecturing to a more critical and significant learning based on competencies and not merely on contents. After justifying the need for an experience of this type and describing its development, contents, and evaluation, the article goes on to present the results of the descriptive study which has accompanied it. The outcomes of the investigation have revealed that the ECTS seminar system has been highly beneficial for the participating students. The latter have been aware of the existence, development, and usefulness of a set of generic and specific competencies which are essential for the English philologist. We consider that, in our immediate context, these ECTS seminars have constituted an important first step in the integration, teaching, and assessment of competencies in the curriculum.
\end{abstract}

Keywords: competencies; ECTS; EHEA; cross-curricular; communicative competence.

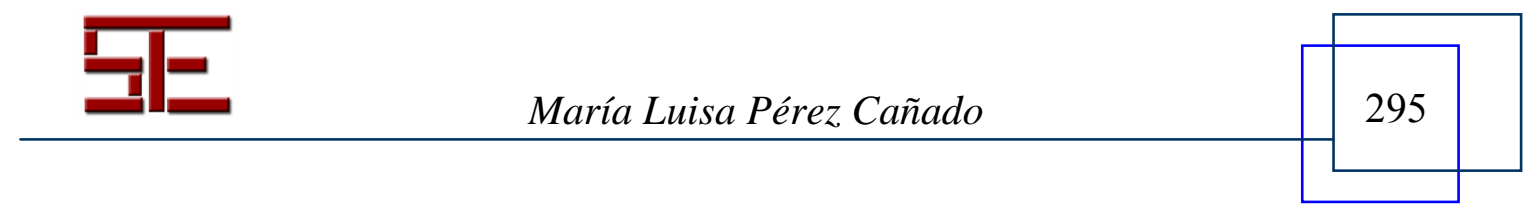




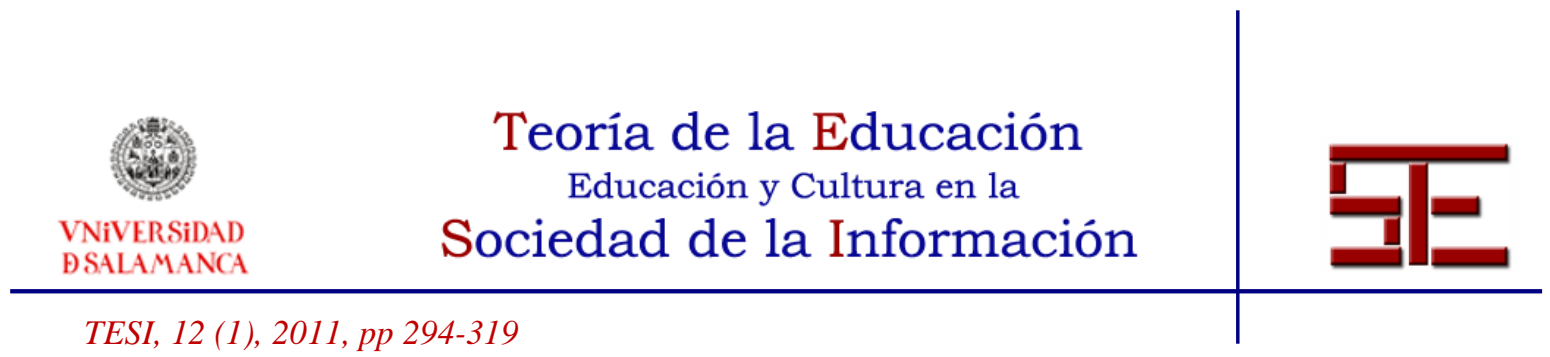

\section{EL DESARROLLO DE COMPETENCIAS COMUNICATIVAS A TRAVÉS DE SEMINARIOS TRANSVERSALES ECTS: UNA EXPERIENCIA EN LA UNIVERSIDAD DE JAÉN}

Fecha de recepción: 27/09/2010; fecha de aceptación: 06/01/2011; fecha de publicación: 31/03/2011

María Luisa Pérez Cañado

mlperez@ujaen.es

Universidad de Jaén

\section{1.- INTRODUCCIÓN}

Actualmente estamos viviendo un proceso de profunda revisión y cambio en la educación terciaria en general y en la enseñanza universitaria de lenguas en particular, tanto en Europa (a través del llamado Proceso de Bolonia) como en Estados Unidos (impulsado por los informes y artículos de la Modern Language Association). En ambos continentes, estamos asistiendo a una revitalización de la enseñanza lingüística en el nivel universitario que supone una intensa renovación curricular y subraya la necesidad de desarrollar competencias para formar a profesionales que pueden incorporarse a la sociedad como ciudadanos activos y útiles.

En Europa y, muy especialmente, en España, este cambio de paradigma educativo es particularmente sustantivo, ya que conlleva la transformación de la función del docente y del aprendiz, así como un cambio drástico del sistema de enseñanza y aprendizaje. En nuestro país, ha primado tradicionalmente la enseñanza como transmisión del conocimiento y el aprendizaje como reproducción, lo que se podría denominar un "aprendizaje bulímico" (Pérez Cañado, en prensa), donde el alumnado recibe ingentes cantidades de datos, los memoriza y reproduce en el examen, para olvidarlos posteriormente por su falta de actualidad o de aplicabilidad a nuevos contextos.

Ahora, todos los datos que previamente acumulaba el aprendiz están accesibles en las redes de información y lo importante y necesario es enseñar al estudiante a saber buscarlos, localizarlos, interpretarlos y utilizarlos adecuadamente. Se ha de evolucionar del aprendizaje por asociación o conocimiento pragmático al aprendizaje comprensivo, significativo y relevante (Pérez Gómez et al., 2009c); del conocimiento superficial al conocimiento selectivo y profundo (Pérez Gómez et al., 2009d); de los contenidos (programas) a los resultados del aprendizaje (competencias y descriptores) (Pérez

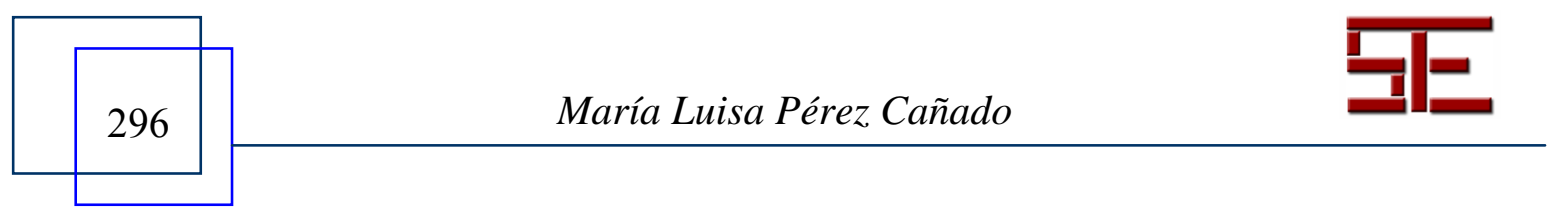




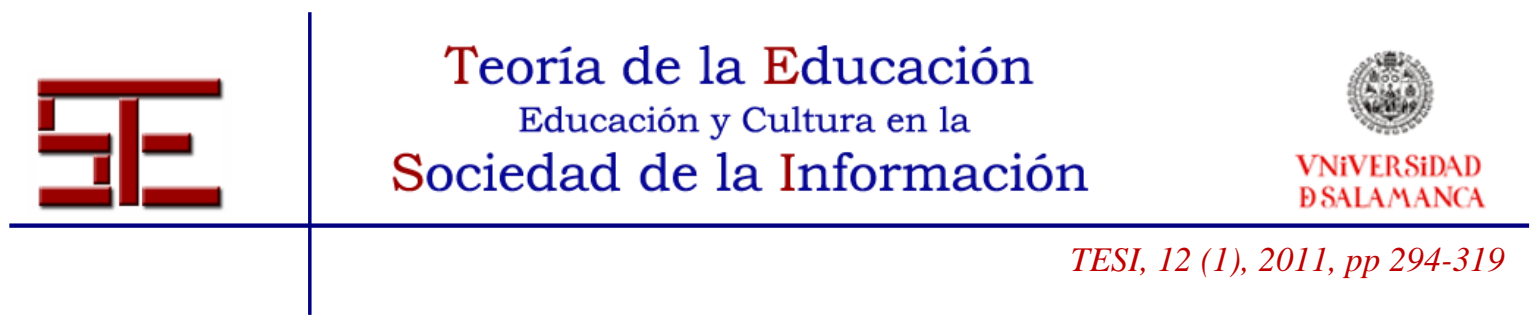

Gómez et al., 2009a). En definitiva, se ha de realizar la transición desde la cultura tradicional de la enseñanza racionalista, memorista y transmisiva hacia la cultura del aprendizaje permanente (Junta de Andalucía, 2009a).

Claves en la consecución de este objetivo son las competencias. Éstas no sólo incluyen conocimientos, sino también habilidades, destrezas, actitudes y valores (OECD, DeSeCo, 2003). Como señala la Junta de Andalucía (Pérez Gómez et al., 2009e: 9), "El conocimiento universitario aprendido en contextos académicos exclusivamente de reproducción no desarrolla competencias, cuesta mucho aprenderlo y poco olvidarlo". Las competencias constituyen el marco de referencia para la selección de contenidos e implican no sólo "saber", sino "saber hacer" y "querer hacer" en contextos y situaciones concretas (Pérez Gómez et al., 2009a:18). En este sentido, las competencias promueven la conexión con el mundo laboral y con los contextos y situaciones que los alumnos tendrán que afrontar en su vida personal, social y profesional: "El concepto de competencia representa una apuesta decidida por acercar el aprendizaje a los problemas y exigencias de la vida contemporánea" (Junta de Andalucía, 2009a:14).

A pesar de su importancia, las competencias rara vez se han incorporado de manera explícita al currículo. Como señala Martín Ortega (2008), no se enseñan en la universidad, sino que se consideran subproductos de la enseñanza de contenidos. Esta situación ha de superarse incorporando las competencias -en especial, las transversales genéricas- explícitamente al currículo, ya que, de lo contrario, corremos el riesgo de que no se trabajen durante los estudios universitarios.

Estamos precisamente en el momento idóneo para incorporar las competencias al currículo y a los nuevos planes de estudio, ya que, como señala la Declaración de Graz (2003:5), tenemos que hacer frente al reto de la adaptación metodólogica al EEES y pasar de la teoría a la práctica: the main challenge now is to transform the multitude of legislative changes that have been taking place across Europe in the past few years into meaningful academic aims and institutional realities. Hemos alcanzado el máximo nivel de saturación teórica en la formulación de competencias y ahora hay que proporcionar concreciones prácticas que orienten al profesorado en su actividad docente. Se ha de pasar de su formulación teórica a su concreción en el currículo (Pérez González, 2009: 94): "It appears that this new way of understanding educational objectives has reached its maximum level of theoretical saturation and has produced considerable theoretical reflections. However, practical specifications are needed to guide teachers in their

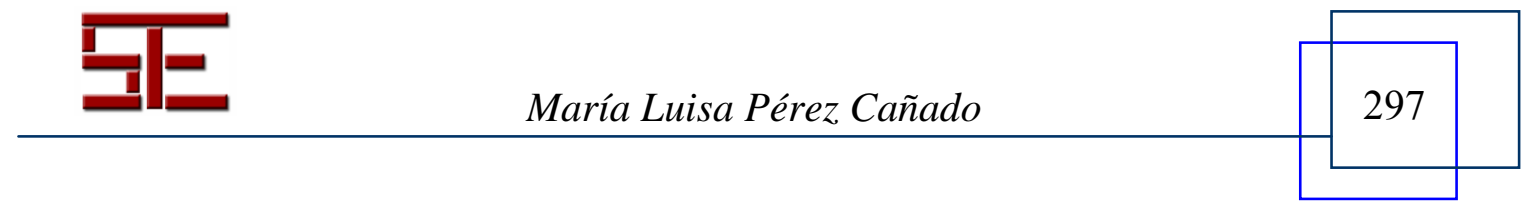




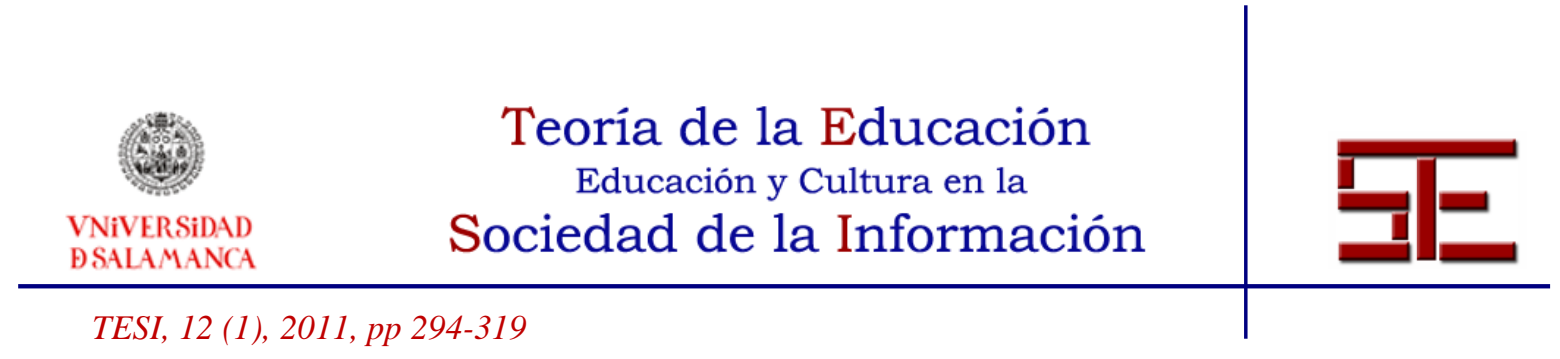

professional activity. [...] The move needs to be made from their theoretical formulation to their concretion in the educational curriculum".

Con esta convicción de la necesidad de incorporar las competencias de manera práctica y explícita en el currículo, hemos diseñado e implantado un novedoso sistema de seminarios transversales para alumnado en la Licenciatura de Filología Inglesa de la Universidad de Jaén durante el curso académico 2008-2009. Estos seminarios entroncan, de una parte, con el Plan de Acción Tutorial (PAT) de la titulación y, de otra, con el pilotaje del sistema ECTS (European Credit Transfer system) en la misma.

El presente artículo comienza justificando y describiendo este sistema de seminarios y presenta los resultados obtenidos en las sesiones dedicadas a desarrollar las competencias de comunicación oral y escrita, implementadas durante el primer cuatrimestre del presente curso académico. Se concluye examinando las dificultades y ventajas de esta novedosa manera de trabajar las competencias y valorando la experiencia en su conjunto.

\section{2.- DESCRIPCIÓN DE LA EXPERIENCIA}

\section{1.- Justificación y objetivos de los seminarios}

Son muchas las razones que justifican el sistema de seminarios proyectado. En primer lugar, está avalado por los resultados recientemente obtenidos en un estudio diagnóstico del funcionamiento del crédito europeo en la Licenciatura de Filología Inglesa de la Universidad de Jaén (véase Pérez Cañado y Casas Pedrosa, en prensa). Los más de 200 alumnos entrevistados señalaron unánimemente las carencias en inglés instrumental (comunicación oral y escrita en lengua inglesa) como la mayor laguna en la titulación en cuestión. Por tanto, urgía su desarrollo explícito de modo interdisciplinar y transversal.

De otra parte, tal y como se señala en la bibliografía más reciente sobre adaptación al Espacio Europeo (Junta de Andalucía, 2009b y 2009d), las enseñanzas de grado han de garantizar el desarrollo óptimo de las habilidades de comunicación oral y escrita de los universitarios: "Las competencias fundamentales a adquirir en los nuevos títulos de grado incluyen la comunicación ágil y clara, oral y escrita, utilizando los recursos TIC, al menos en la lengua materna y en una segunda lengua de ámbito internacional" (Pérez Gómez et al., 2009b: 6). En este sentido, consideramos que, mientras que los contenidos puramente teóricos están al alcance de un ratón en las redes de información, la

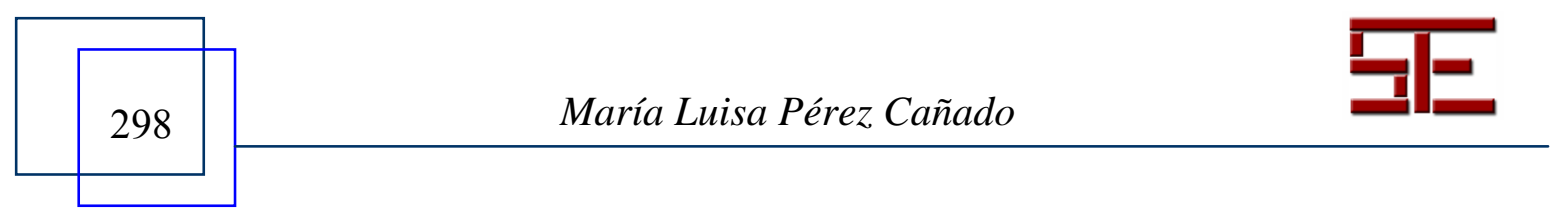




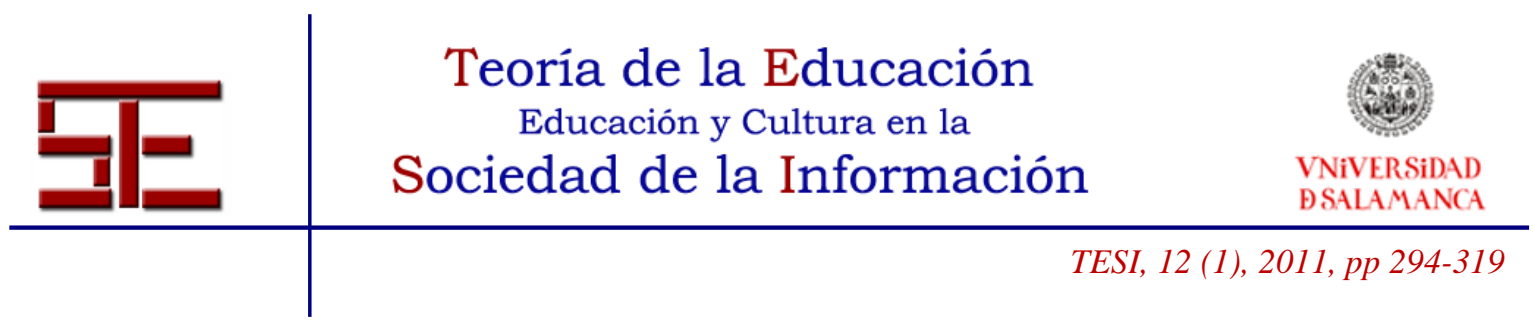

competencia oral y escrita no se puede improvisar; se ha de enseñar y trabajar, ya que se corresponde directamente con las necesidades de los ciudadanos para incorporarse a la sociedad de modo activo y útil en cualquier contexto académico.

Precisamente para nuestro alumnado concreto, estas competencias se han desvelado como especialmente problemáticas (quizás debido a esa laguna anteriormente diagnosticada), ya que venimos constatando su falta de dominio del inglés oral y serios escollos con el plagio en su inglés escrito. Aprovechando que en el presente curso académico se ha puesto en marcha el PAT de la Universidad de Jaén, que pretende "guiar a los alumnos tutelados en el desarrollo de sus estrategias de aprendizaje para la mejora de su rendimiento académico" y "capacitar a los universitarios para ser futuros profesionales íntegros, responsables y eficaces", se ha programado atacar estas deficiencias a través del sistema de seminarios que aquí describimos.

Por último, y dado que también se relaciona directamente con el pilotaje del sistema ECTS en la titulación de Filología Inglesa, se ha pretendido mejorar por medio de él la interdisciplinariedad, la coordinación entre profesores, la superación del individualismo que a menudo se considera caracteriza a las actuaciones docentes del profesorado universitario (Zabalza, 2004) y la creación de equipos docentes, tan importantes en la nueva filosofía subyacente al EEES (Michavila, 2007).

\section{2. - Caracterización y organización de los seminarios ECTS}

Con estos fines, se ha diseñado un programa de seminarios que se ha desarrollado en los meses lectivos del presente curso académico. Se trata de una de las tres maneras en que estamos integrando explícitamente las competencias en nuestra titulación (Poblete, 2008). Las otras dos son trabajarlas a tres niveles de dominio en las asignaturas concretas, en progresión por cursos, e incluirlas en un curso de pregrado que se impartirá en la Universidad de Jaén en octubre de 2009 con el fin de dotar al alumnado de las técnicas que necesita para afrontar con éxito la nueva metodología que supone en Espacio Europeo de Educación Superior (EEES). Ha habido tres seminarios programados al cuatrimestre (seis, por tanto, a lo largo del año académico), que se han ubicado en un mismo día para cada ciclo, de tal modo que el alumnado de primer ciclo ha asistido a dichos seminarios los martes de cada mes lectivo, y el de segundo ciclo, los miércoles (véase Fig. 1).

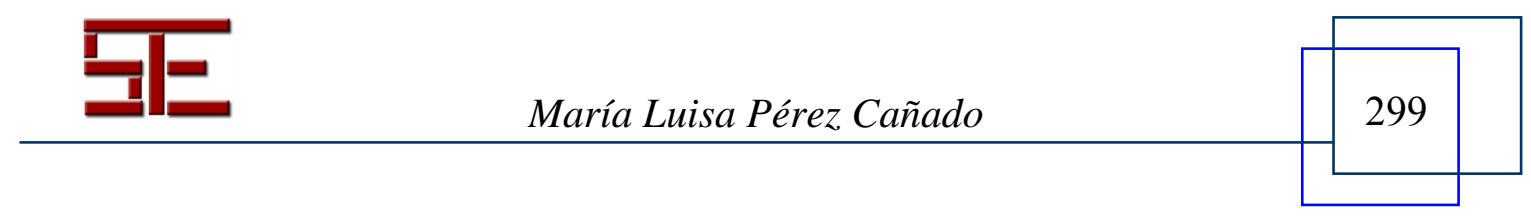



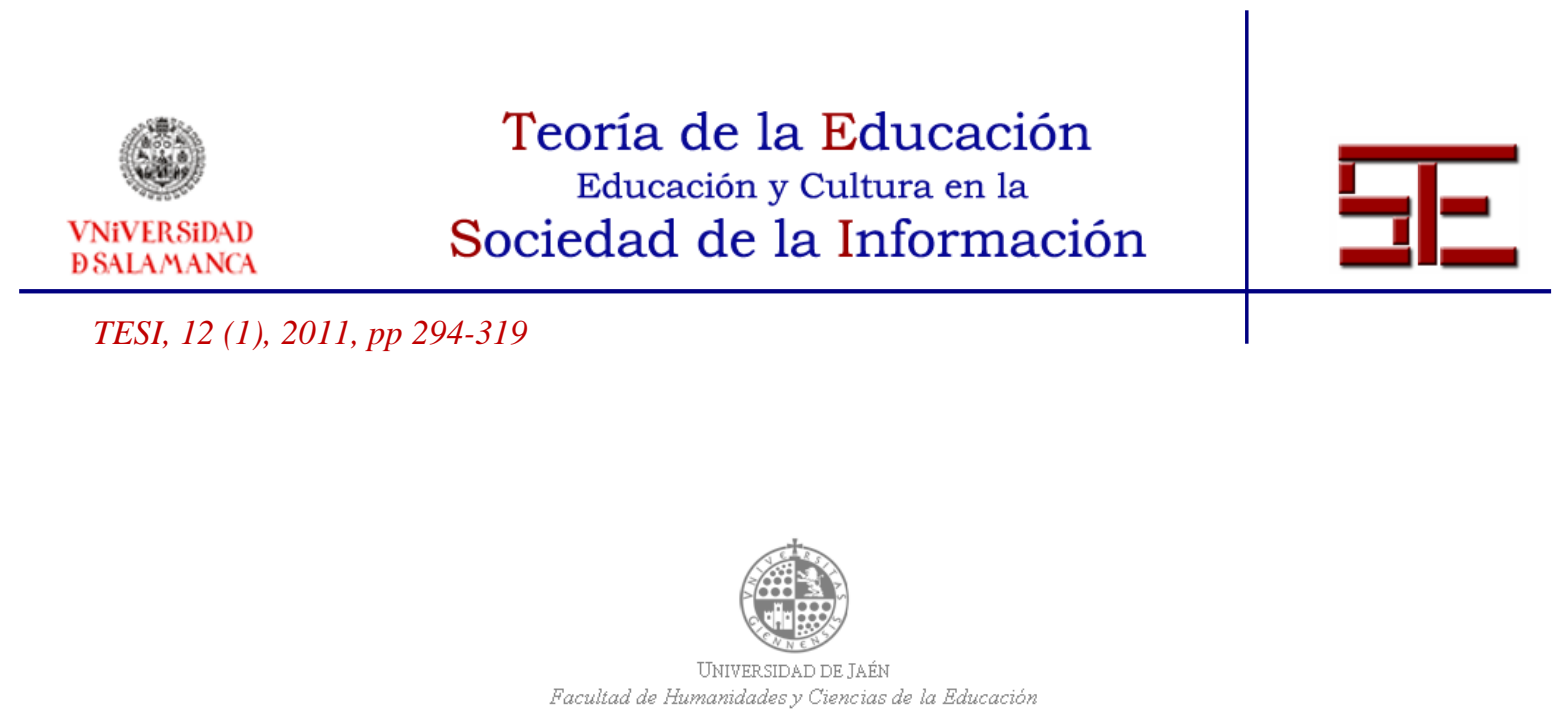

Facultad de Humanidades y Ciencias de la Educación

CURSO ACADÉMICO 2008/09: HORARIO DE ASIGNATURAS TRONCALES Y OBLIGAT ORIAS / OPTATIVAS

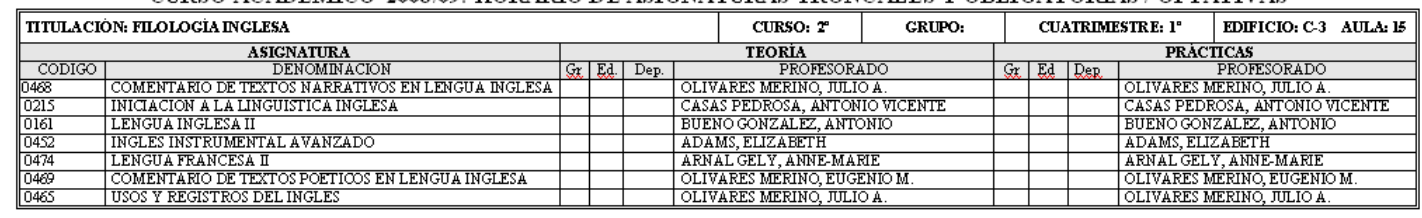

\begin{tabular}{|c|c|c|c|c|c|}
\hline $1030-11: 30$ & & & Lengua Inzlesa I (P) & & \\
\hline $11: 00-12: 30$ & & & Lengua Inqlesa I (P) & & \\
\hline $12: 30-13: 30$ & & & Inglés Instrumental Avranzado (P) & & \\
\hline $13: 30-14: 30$ & & & Inqlés Intrumental Avanzado (P) & & \\
\hline HORA/DIA & LUNES & MARTES & MIERR COLES & JUEVES & VIERNES \\
\hline $1530-16: 30$ & $\begin{array}{l}\text { Comentario de Textos Narratives en } \\
\text { Lengua Inglesa }\end{array}$ & Lengun Frances:a II $(M)$ & 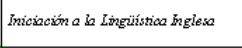 & Lengus Francessa II & $\begin{array}{l}\text { Comentario de Textos Póticos en } \\
\text { Lengua Inglesc }\end{array}$ \\
\hline $1630-17: 30$ & $\begin{array}{l}\text { Comentario de Texos Norratiog fon } \\
\text { Lengua Inglesa (F) }\end{array}$ & Usos y Registros del higlés $(S)$ & Tengua Inglesa II & Lengua Francesa II (F) & $\begin{array}{l}\text { Comentario de Textos Póticos en } \\
\text { Lengua Inglesa }(P)\end{array}$ \\
\hline $17: 00-18: 30$ & Usos y Registros del higlés & $\begin{array}{l}\text { Comentario de Texos Póticos en Lengua } \\
\text { Inglesa (N) }\end{array}$ & 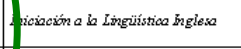 & Lengua Inglesa II & Lengua Inzlesa II (S) \\
\hline $18: 00-19: 30$ & Usos y Registros del higles (R) & 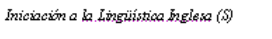 & rqlés Instrumertal Avanzado & Inqlés Instrumertal Av anzzado & Inqlés Intrumental Avanzado (S) \\
\hline $19: 30-20: 30$ & $\begin{array}{l}\text { Comentario de Texas Poéticosen } \\
\text { Lengua Inglesc }\end{array}$ & $\begin{array}{l}\text { Comentario de Textos Norrativos en Le } \\
\text { Inglesa }(M)\end{array}$ & $\begin{array}{l}\text { Comentario de Textos Nurrativos en } \\
\text { Lengua Inglesa }\end{array}$ & & \\
\hline $2030-21: 30$ & 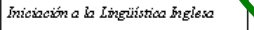 & 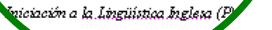 & Usos y Registros del haglés & & \\
\hline
\end{tabular}

Figura 1. Organización de las horas semanales dedicadas al grupo de trabajo (seminario) en primer ciclo.

En estas sesiones monográficas, se han trabajado de forma transversal e interdisciplinar una serie de temas que se han detectado como especialmente problemáticos para el alumnado de la titulación en cuestión (e. g. ensayos académicos y plagio), que previamente se cubrían de forma individual y heterogénea en diversas asignaturas de un mismo módulo y que desarrollan las competencias genéricas y específicas identificadas como centrales para el filólogo inglés. A través del programa de seminarios, el profesorado que previamente trataba dichos temas de forma inconexa ha unificado criterios y trabajado de forma coordinada para presentar ese tema transversal de modo consensuado y unificado en la sesión monográfica. El material elaborado específicamente por el equipo docente se ha colgado en la plataforma ILIAS para que el profesorado y alumnado de los cuatro cursos de Filología Inglesa puedan acceder a él y comentarlo a través del foro de discusión creado en dicho entorno virtual (véase Fig. 2).

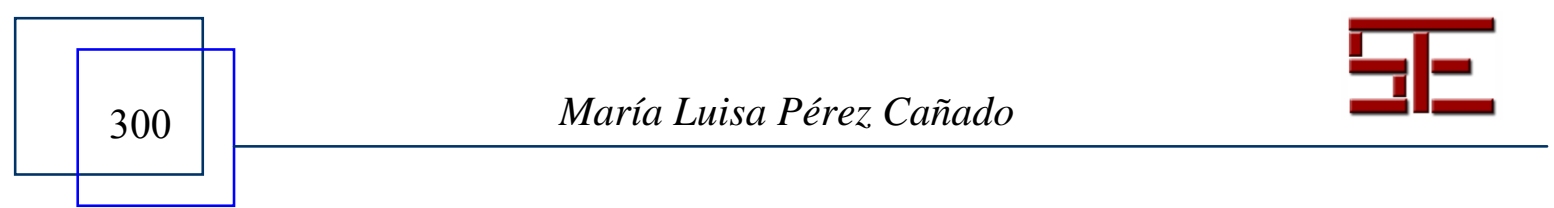




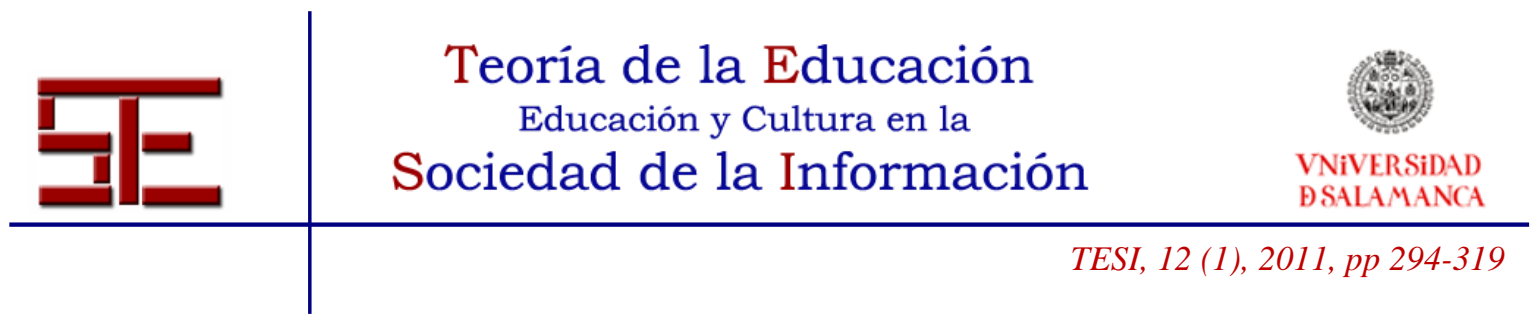

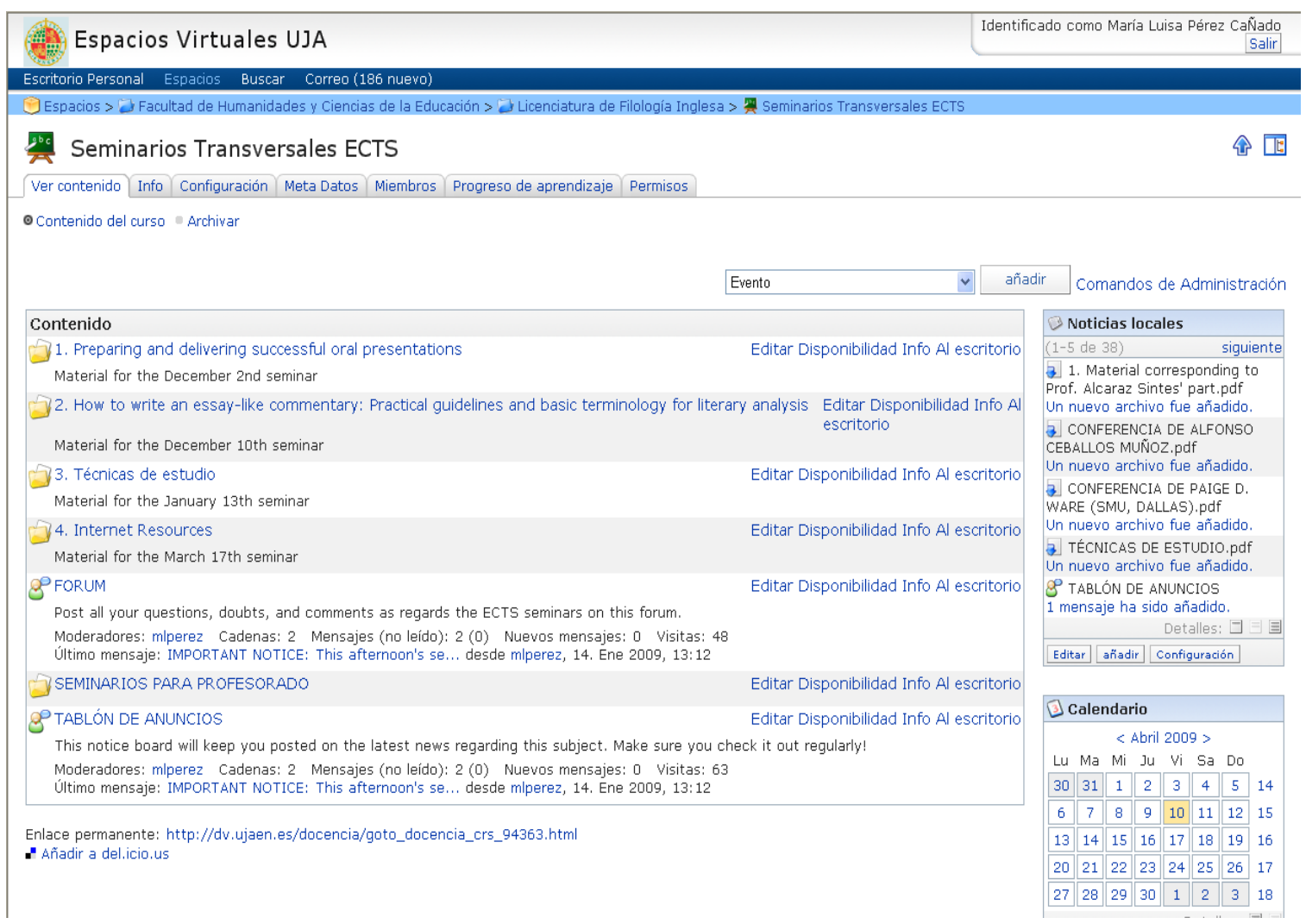

Figura 2. Espacio dedicado a los seminarios en la plataforma vitual ILIAS.

Aunque no es objetivo del presente artículo, también nos gustaría destacar que se ha desarrollado simultáneamente un sistema de seminarios para profesorado, que ha coincidido en calendario con el desarrollado para el alumnado. Se han organizado seis sesiones -aproximadamente una por cada mes lectivo- en las que el profesorado de la titulación comparte, a través de ponencias de una hora, las experiencias docentes que está llevando a cabo en su asignatura. Se ha invitado, igualmente, a profesorado de otras universidades españolas, europeas y norteamericanas a que participe en dichas sesiones, exponiendo así experiencias relevantes de innovación pedagógica. El debate e intercambio de opiniones se ha favorecido en cada una de ellas. El fin último de estas sesiones ha sido fomentar la comunicación y colaboración entre el profesorado, permitir la incorporación inmediata en el aula de las buenas prácticas de los demás y activar el desarrollo de proyectos docentes comunes entre equipos de profesores.

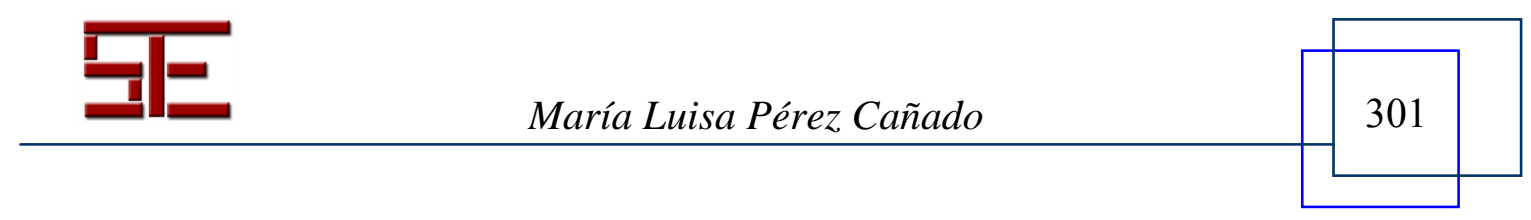




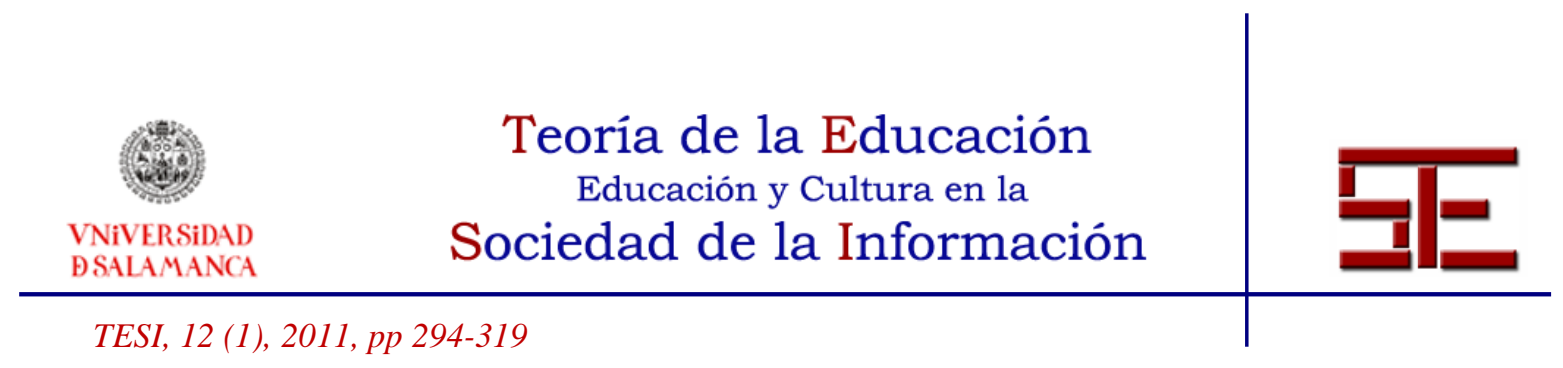

\section{3. - Contenidos de los seminarios ECTS}

Al tratarse de lo que se denomina en la bibliografía especializada "módulos interdisciplinares" o "transversales" (Junta de Andalucía, 2009d), los seminarios ECTS han pretendido desarrollar competencias transversales genéricas y específicas comunes a todas las disciplinas de la titulación, tales como comunicación oral y escrita (a través de exposiciones orales, ensayos literarios y académicos y plagio); habilidades básicas de manejo del ordenador (con sesiones sobre la utilización de diccionarios electrónicos y herramientas, programas y aplicaciones informáticas específicas); habilidad para trabajar de forma autónoma (por medio de seminarios sobre técnicas de estudio); o capacidad para identificar problemas y temas de investigación y evaluar su relevancia (con una sesión de iniciación a la investigación). Se reproduce a continuación el desglose de contenidos y ciclos y la temporización de los seminarios.

\begin{tabular}{|c|c|c|}
\hline$\underline{\text { TEMA DEL SEMINARIO }}$ & $\underline{\text { CICLO }}$ & FECHA \\
\hline $\begin{array}{l}\text { Preparing and delivering successful } \\
\text { oral presentations }\end{array}$ & $1^{o}$ & 2 de diciembre de 2008 \\
\hline $\begin{array}{c}\text { How to write an } \\
\text { essay-like commentary: Practical } \\
\text { guidelines and basic terminology for } \\
\text { literary } \\
\text { analysis }\end{array}$ & $2^{\circ}$ & 10 de diciembre de 2008 \\
\hline Técnicas de estudio & $1^{o}$ & 13 de enero de 2009 \\
\hline Ensayos académicos y plagio & $2^{\circ}$ & 14 de enero de 2009 \\
\hline Recursos de Internet & $\mathbf{1}^{\mathbf{0}}$ & 17 de marzo de 2009 \\
\hline Recursos de Internet & $2^{\circ}$ & 18 de marzo de 2009 \\
\hline Ensayos académicos y plagio & $\mathbf{1}^{\mathbf{0}}$ & 21 de abril de 2009 \\
\hline $\begin{array}{l}\text { Utilización de diccionarios } \\
\text { electrónicos }\end{array}$ & $2^{\circ}$ & 22 de abril de 2009 \\
\hline
\end{tabular}

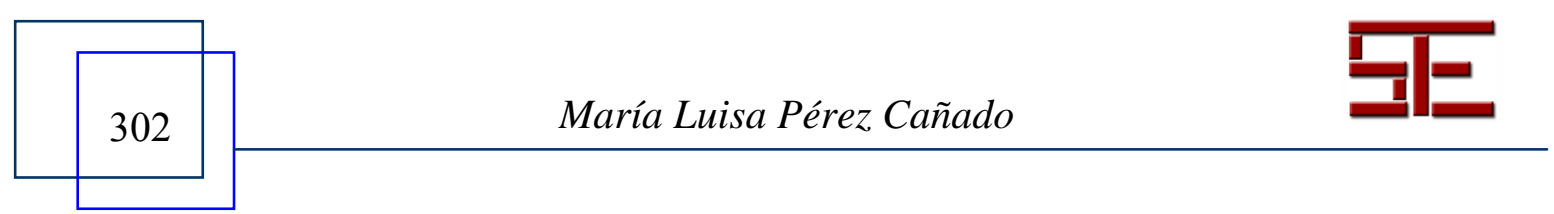




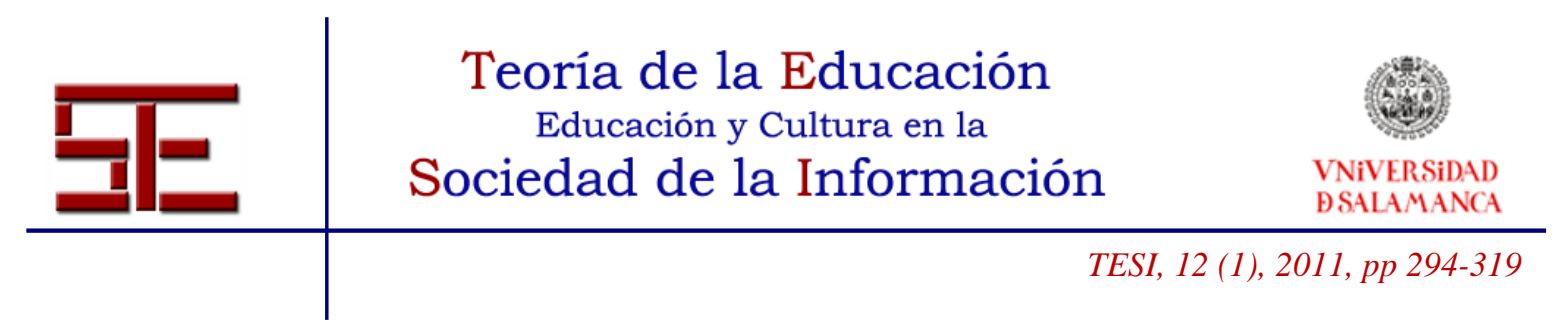

\begin{tabular}{|c|c|c|}
\hline $\begin{array}{c}\text { Introducción a la pronunciación del } \\
\text { inglés (nivel B2) }\end{array}$ & $1^{\mathbf{0}}$ & 12 de mayo de 2009 \\
\hline Iniciación a la investigación & $2^{\circ}$ & 13 de mayo de 2009 \\
\hline
\end{tabular}

Tabla 1. Sistema de seminarios para alumnado curso 2008-2009.

En este artículo, nos centraremos en los tres seminarios que han desarrollado la competencia oral y escrita en inglés: los correspondientes a exposiciones orales (diciembre de 2008), ensayos literarios (diciembre de 2008) y ensayos académicos y plagio (enero de 2009). El primero se ha desarrollado con alumnado de primer ciclo de Filología Inglesa y los dos últimos, con alumnado de segundo ciclo. Los tres se han celebrado en el primer cuatrimestre del presente curso académico (2008-2009), a lo largo de 6 horas lectivas (15.30 a 21.30).

\section{4. - Evaluación de los seminarios}

Por último, hay que destacar que, para valorar las dificultades y el éxito de la iniciativa, se han diseñado y validado diversos instrumentos de recogida de información. Entre ellos, hay que destacar, en primer lugar, una Activity Feedback Form, donde el alumnado ha resumido cualitativamente en preguntas abiertas los objetivos, contenidos y conclusiones de cada seminario monográfico, ofreciendo asimismo su opinión acerca del mismo (véase Apéndice I). También ha cumplimentado un cuestionario con escala Likert de 4 puntos para valorar los objetivos, contenidos, impartición y evaluación de cada sesión, así como las principales competencias genéricas y específicas que considera se han desarrollado (véase Apéndice II). Asimismo, se ha mantenido un control de asistencia en cada sesión, ya que los seminarios han tenido lugar en horario lectivo y en sustitución de las clases regulares.

Estos instrumentos son los que nos han permitido obtener los resultados del funcionamiento de la experiencia que detallamos en la siguiente sección.

\section{3. - EL ESTUDIO}

\section{1. - Objetivos}

Son tres los objetivos principales que hemos perseguido con el presente estudio y que formulamos a continuación a través de las siguientes hipótesis:

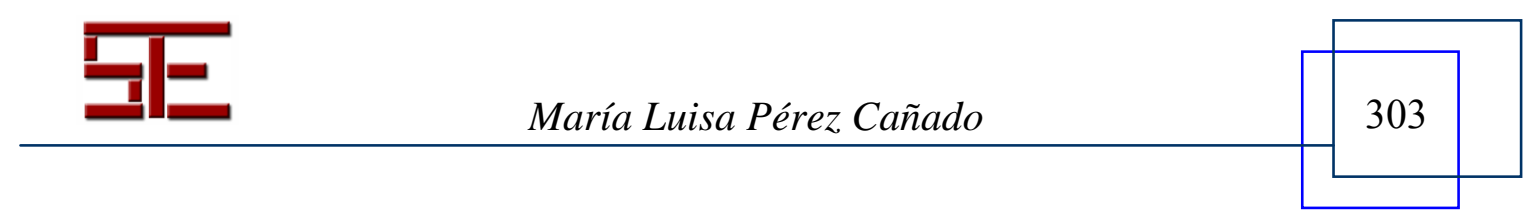




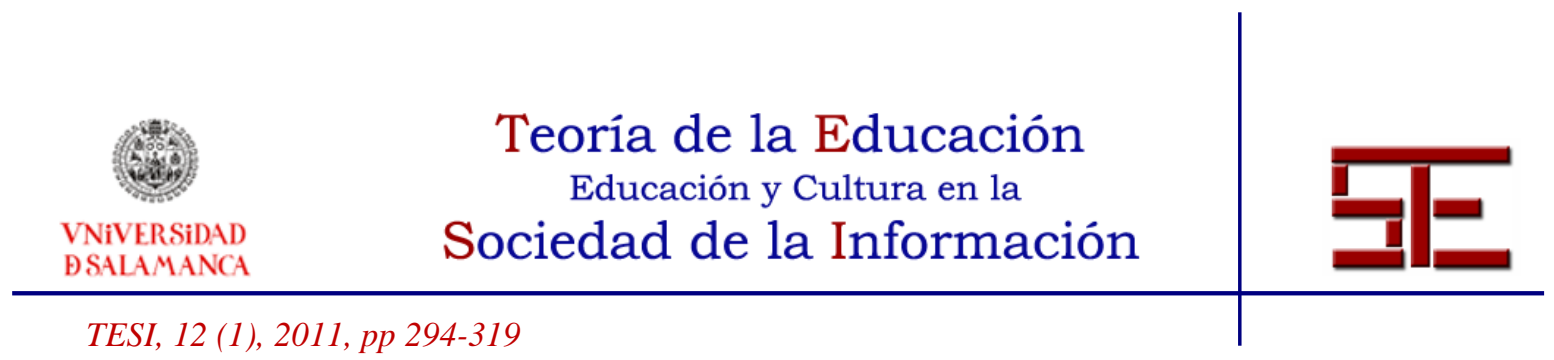

1. Los seminarios ECTS para el desarrollo de la competencia oral y escrita se han considerado adecuados y útiles en todos sus niveles de desarrollo.

2. Los seminarios ECTS han proporcionado al alumnado las herramientas idóneas para realizar exposiciones orales y ensayos literarios y académicos en inglés, y para evitar el plagio.

3. Los seminarios ECTS han permitido el desarrollo de competencias genéricas y específicas identificadas como clave para el filólogo inglés (véase Libro Blanco Título de Grado en Estudios en el Ámbito de la Lengua, Literatura, Cultura y Civilización de la ANECA).

\section{2. - Instrumentos y variables}

Los instrumentos utilizados para determinar la consecución de los precitados objetivos han sido los dos descritos en el apartado de evaluación (2.4.). Se ha tratado de un cuestionario con escala Likert de 4 puntos y otro abierto (Activity Feedback Form), donde el alumnado ha reflexionado sobre el funcionamiento de los seminarios y el desarrollo de competencias. Las variables consideradas han sido las siguientes:

1. Aspectos del seminario:

- El tema del seminario ha sido útil.

- Los objetivos del seminario han estado claramente establecidos.

- Los contenidos del seminario han sido útiles.

- Los materiales del seminario se han preparado y presentado adecuadamente.

- La impartición del seminario ha sido adecuada.

- Los materiales para evaluar el seminario han sido adecuados.

- Este seminario ha proporcionado a los asistentes las herramientas idóneas para realizar exposiciones orales en inglés/ensayos literarios/ensayos académicos con confianza.

2. Competencias genéricas desarrolladas:

- Capacidad de análisis y síntesis.

- Capacidad de aplicar los conocimientos en la práctica.

- Conocimientos generales básicos sobre el área de estudio.

- Capacidad de aprender. 


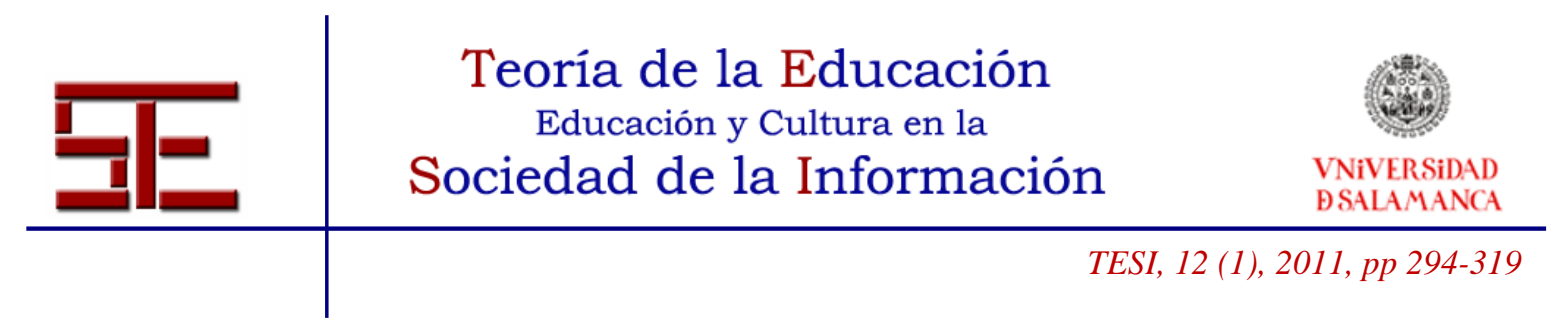

- Capacidad crítica y autocrítica.

- Capacidad para generar nuevas ideas (creatividad).

- Toma de decisiones.

- Trabajo en equipo.

- Habilidad para trabajar de forma autónoma.

- Compromiso ético.

3. Competencias específicas desarrolladas:

- Dominio instrumental de la lengua inglesa.

- Capacidad de comunicación oral y escrita en lengua inglesa.

- Capacidad para comunicar y enseñar los conocimientos adquiridos.

- Capacidad de comunicación y perlocutiva.

- Capacidad de análisis y síntesis de documentación compleja.

- Conocimiento de retórica estilística.

- Conocimiento de crítica textual y edición de textos.

\section{3. - Muestra}

Hemos trabajado con un total de 36 alumnos, 31 en primer ciclo y 5 en segundo (se trata de la práctica totalidad del alumnado matriculado en la Licenciatura en Filología Inglesa). El desglose por curso y género se presenta en la siguiente tabla:

\begin{tabular}{|c|c|c|c|}
\hline & ALUMNOS & ALUMNAS & TOTAL \\
\hline $1^{\circ}$ DE FILOLOGÍA & 5 & 14 & 19 \\
\hline \multicolumn{4}{|l|}{ INGLESA } \\
\hline $2^{\circ}$ DE FILOLOGÍA & 5 & 7 & 12 \\
\hline \multicolumn{4}{|l|}{ INGLESA } \\
\hline $3^{\circ}$ DE FILOLOGÍA & 1 & --- & 1 \\
\hline \multicolumn{4}{|l|}{ INGLESA } \\
\hline $4^{\circ}$ DE FILOLOGÍA & 1 & 3 & 4 \\
\hline \multicolumn{4}{|l|}{ INGLESA } \\
\hline TOTAL & 12 & 24 & 36 \\
\hline
\end{tabular}

Tabla 2. Muestra.

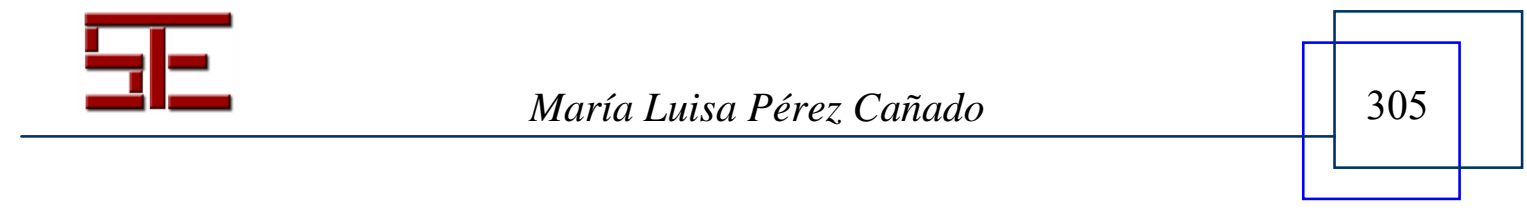




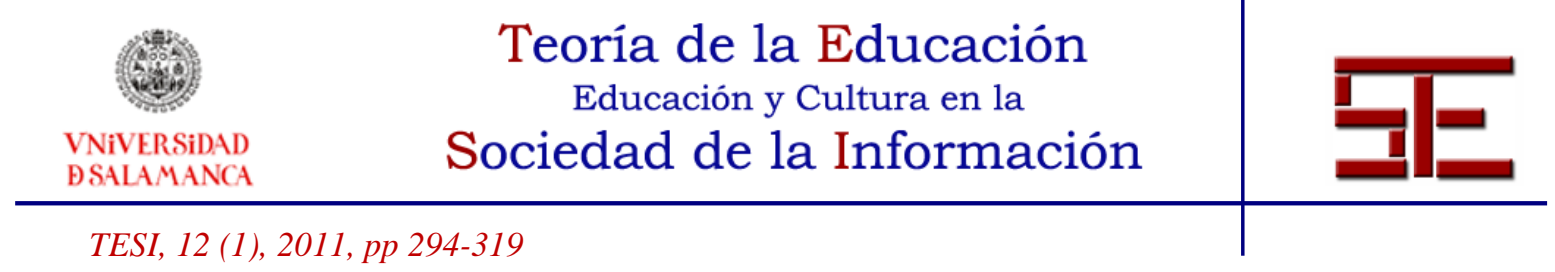

\section{4. - Metodología estadística}

Para el análisis de los resultados, hemos utilizado estadística descriptiva, empleando el programa SPSS en su versión 16.0. Hemos hecho uso de la media, mediana, moda, desviación típica, máximo y mínimo.

\section{5. - Resultados}

\subsection{1. - Valoración de los seminarios}

Lo primero que constatamos es que los tres seminarios reciben una altísima valoración por parte del alumnado en todos los aspectos de su desarrollo (véanse Tablas 3, 4 y 5).

\begin{tabular}{|c|ccccccc|}
\hline $\begin{array}{c}\text { EXPOSICIONES } \\
\text { ORALES }\end{array}$ & $\mathrm{P} 1$ & $\mathrm{P} 2$ & $\mathrm{P} 3$ & $\mathrm{P} 4$ & $\mathrm{P} 5$ & $\mathrm{P} 6$ & $\mathrm{P} 7$ \\
\hline N & $\mathbf{3 1}$ & $\mathbf{3 1}$ & $\mathbf{3 1}$ & $\mathbf{3 1}$ & $\mathbf{3 1}$ & $\mathbf{3 1}$ & $\mathbf{3 1}$ \\
\hline Media & $\mathbf{3 , 6 5}$ & $\mathbf{3 , 6 2}$ & $\mathbf{3 , 8 5}$ & $\mathbf{3 , 7 3}$ & $\mathbf{3 , 5 8}$ & $\mathbf{3 , 8 1}$ & $\mathbf{3 , 7 3}$ \\
\hline Mediana & $\mathbf{4}$ & $\mathbf{4}$ & $\mathbf{4}$ & $\mathbf{4}$ & $\mathbf{4}$ & $\mathbf{4}$ & $\mathbf{4}$ \\
\hline Moda & $\mathbf{4}$ & $\mathbf{4}$ & $\mathbf{4}$ & $\mathbf{4}$ & $\mathbf{4}$ & $\mathbf{4}$ & $\mathbf{4}$ \\
\hline Desv. típ. & $\mathbf{0 , 4 9}$ & $\mathbf{0 , 5}$ & $\mathbf{0 , 3 7}$ & $\mathbf{0 , 4 5}$ & $\mathbf{0 , 5}$ & $\mathbf{0 , 4}$ & $\mathbf{0 , 4 5}$ \\
\hline Mínimo & $\mathbf{3}$ & $\mathbf{3}$ & $\mathbf{3}$ & $\mathbf{3}$ & $\mathbf{3}$ & $\mathbf{3}$ & $\mathbf{3}$ \\
\hline Máximo & $\mathbf{4}$ & & $\mathbf{4}$ & $\mathbf{4}$ & $\mathbf{4}$ & $\mathbf{4}$ & $\mathbf{4}$ \\
\hline
\end{tabular}

Tabla 3. Valoración del seminario sobre competencia oral.

\begin{tabular}{|cccccccc|}
\hline $\begin{array}{c}\text { ENSAYOS } \\
\text { LITERARIOS }\end{array}$ & P1 & P2 & P3 & P4 & P5 & P6 & P7 \\
\hline N & $\mathbf{5}$ & $\mathbf{5}$ & $\mathbf{5}$ & $\mathbf{5}$ & $\mathbf{5}$ & $\mathbf{5}$ & $\mathbf{5}$ \\
\hline Media & $\mathbf{4}$ & $\mathbf{3 , 8}$ & $\mathbf{4}$ & $\mathbf{3 , 8}$ & $\mathbf{3 , 6}$ & $\mathbf{4}$ & $\mathbf{3 , 8}$ \\
\hline Mediana & $\mathbf{4}$ & $\mathbf{4}$ & $\mathbf{4}$ & $\mathbf{4}$ & $\mathbf{4}$ & $\mathbf{4}$ & $\mathbf{4}$ \\
\hline Moda & $\mathbf{4}$ & $\mathbf{4}$ & $\mathbf{4}$ & $\mathbf{4}$ & $\mathbf{4}$ & $\mathbf{4}$ & $\mathbf{4}$ \\
\hline Desv. típ. & $\mathbf{0}$ & $\mathbf{0 , 4 5}$ & $\mathbf{0}$ & $\mathbf{0 , 4 5}$ & $\mathbf{0 , 5 5}$ & $\mathbf{0}$ & $\mathbf{0 , 4 5}$ \\
\hline Mínimo & $\mathbf{4}$ & $\mathbf{3}$ & $\mathbf{4}$ & $\mathbf{3}$ & $\mathbf{3}$ & $\mathbf{4}$ & $\mathbf{3}$ \\
\hline Máximo & $\mathbf{4}$ & $\mathbf{4}$ & $\mathbf{4}$ & $\mathbf{4}$ & $\mathbf{4}$ & $\mathbf{4}$ & $\mathbf{4}$ \\
\hline
\end{tabular}

Tabla 4. Valoración del seminario sobre competencia escrita: ensayos literarios.

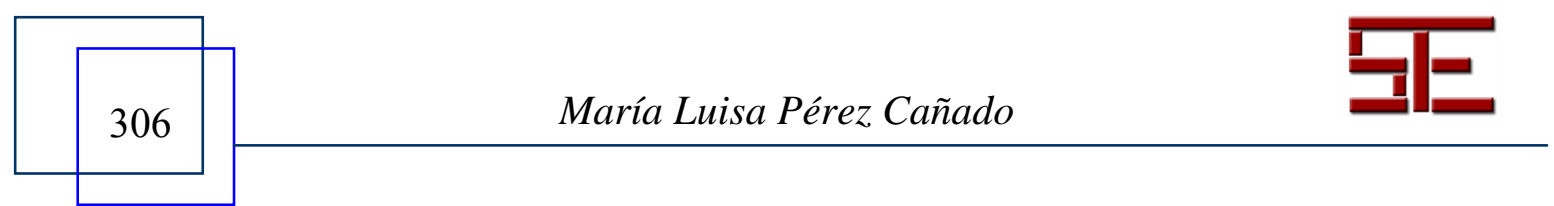




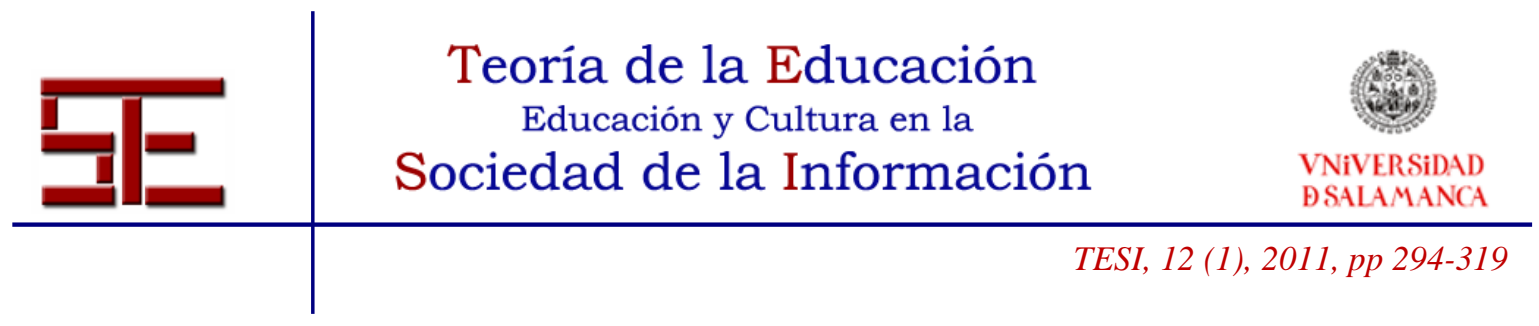

\begin{tabular}{|ccccccccc|}
\hline $\begin{array}{c}\text { ENSAYOS } \\
\text { ACADÉMICOS }\end{array}$ & P1 & P2 & P3 & P4 & P5 & P6 & P7 \\
\hline N & $\mathbf{5}$ & $\mathbf{5}$ & $\mathbf{5}$ & $\mathbf{5}$ & $\mathbf{5}$ & $\mathbf{5}$ & $\mathbf{4}$ & $\mathbf{4}$ \\
\hline Media & $\mathbf{3 , 8}$ & $\mathbf{3 , 4}$ & $\mathbf{3 , 8}$ & $\mathbf{4}$ & $\mathbf{4}$ & $\mathbf{4}$ & $\mathbf{4}$ & $\mathbf{4}$ \\
\hline Mediana & $\mathbf{4}$ & $\mathbf{3}$ & $\mathbf{4}$ & $\mathbf{4}$ & $\mathbf{4}$ & $\mathbf{4}$ & $\mathbf{4}$ \\
\hline Moda & $\mathbf{4}$ & $\mathbf{3}$ & $\mathbf{4}$ & $\mathbf{0}$ & $\mathbf{0}$ & $\mathbf{4}$ & $\mathbf{4}$ & $\mathbf{0}$ \\
\hline Desv. típ. & $\mathbf{0 , 4 5}$ & $\mathbf{0 , 5 5}$ & $\mathbf{3}$ & $\mathbf{3}$ & $\mathbf{4}$ & $\mathbf{4}$ & $\mathbf{4}$ & $\mathbf{4}$ \\
\hline Mínimo & $\mathbf{3}$ & $\mathbf{4}$ & $\mathbf{4}$ & $\mathbf{4}$ & $\mathbf{4}$ & \\
\hline Máximo & $\mathbf{4}$ & &
\end{tabular}

Tabla 5. Valoración del seminario sobre competencia escrita: ensayos académicos.

Las siete variables analizadas han recibido una valoración superior a 3. 5 (sobre 4) en los tres seminarios, lo que indica que el alumnado ha estado muy satisfecho con los objetivos, contenidos, materiales, impartición y evaluación de las sesiones monográficas sobre competencia oral y escrita. Han considerado los temas tratados útiles y opinan que los seminarios les han proporcionado las herramientas necesarias para afrontar con éxito la realización de exposiciones orales y ensayos literarios y académicos en inglés. Los estadísticos mediana y moda confirman los resultados de la media, ya que en prácticamente todos los apartados de los tres seminarios han sido 4 . El mínimo ha sido 3 para todas las sesiones y el máximo, 4. En ningún caso, pues, se ha puntuado con un valor inferior al grado 3 (véase Diagrama 1).

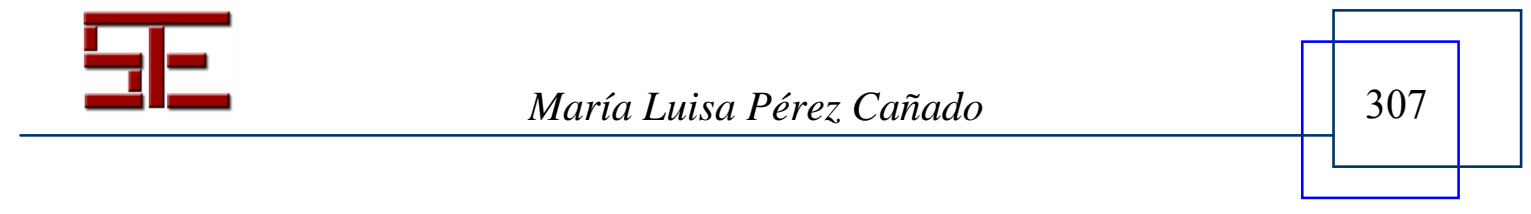



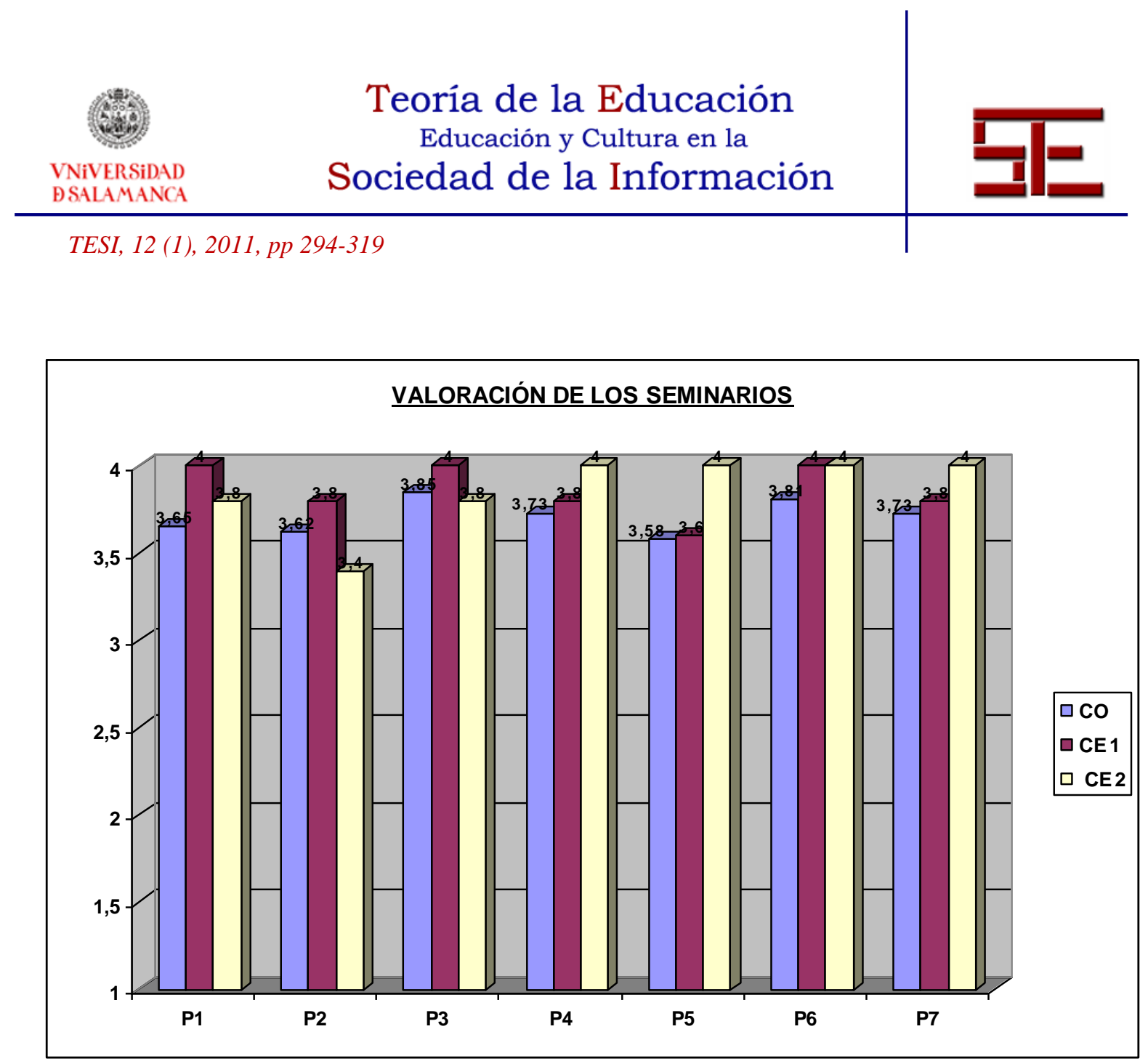

Diagrama 1. Comparación de la valoración de los seminarios.

Esta valoración positiva del desarrollo de los seminarios se ve refrendada por las respuestas del alumnado en las preguntas abiertas del primer instrumento (véase Apéndice I). Sobre el seminario de competencia oral, se ha destacado su utilidad, interés, importancia y carácter práctico. Se reconoce lo positivo de su naturaleza transversal ("They are very important for all subjects") y su enorme utilidad para el futuro académico y profesional del alumnado ("I liked this activity a lot. I think it may help us a lot in our future professional life. And we improve our communicative abilities that help us a lot in our studies"). Solicitan que se repita ("It has been a very good experience. I'd like it to be repeated") y alaban la iniciativa ("Me parece fundamental que la Universidad facilite entrenamiento y formación en competencias transversales como ésta").

A su vez, el seminario sobre ensayos literarios se ha considerado muy enriquecedor, dinámico, práctico interesante y útil: "The seminar has been very interesting and useful

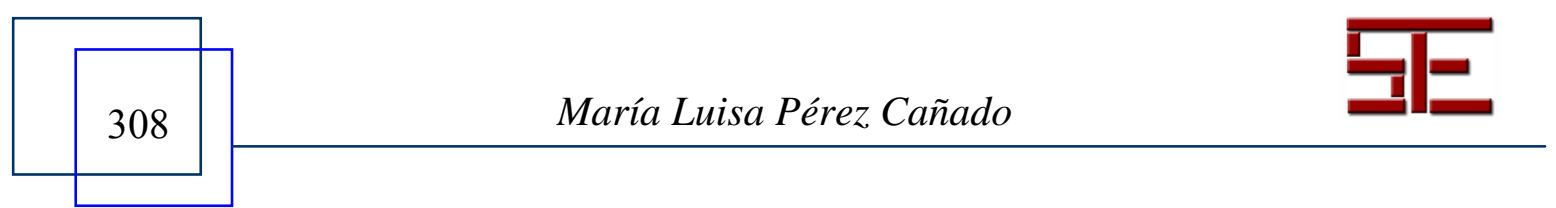




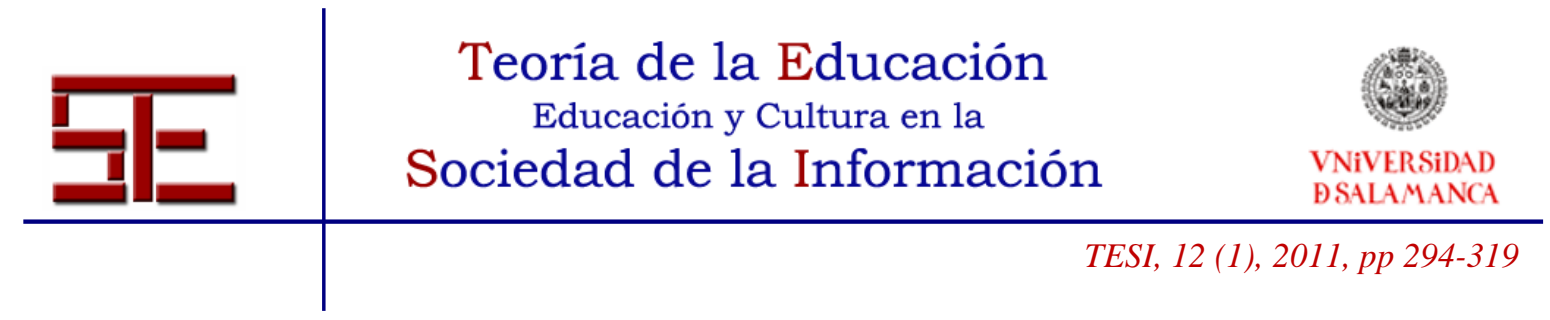

for us to enrich our knowledge of how to write an essay-like commentary and practice on literary analysis".

El correspondiente a ensayos académicos y plagio parece haber sensibilizado al alumnado en este tema que, subraya, nunca había tratado con anterioridad y que considera "extremadamente necesario": "It has been very useful since students are not told about what plagiarism is in any of the subjects". "I believe it was extremely necessary, since plagiarism is unfortunately becoming commonplace (and sometimes without people meaning to)".

\subsection{2. - Competencias genéricas}

Quizás el interés y utilidad manifiestos de los seminarios derive de su potenciación de las competencias. Una vez más, si comparamos las puntuaciones obtenidas en las competencias genéricas, observamos que el alumnado ha considerado en la práctica totalidad de los casos que se desarrollan entre bastante y mucho, ya que, de nuevo, casi todas obtienen entre 3 y 4 puntos sobre un máximo de 4 (véase Diagrama 2).

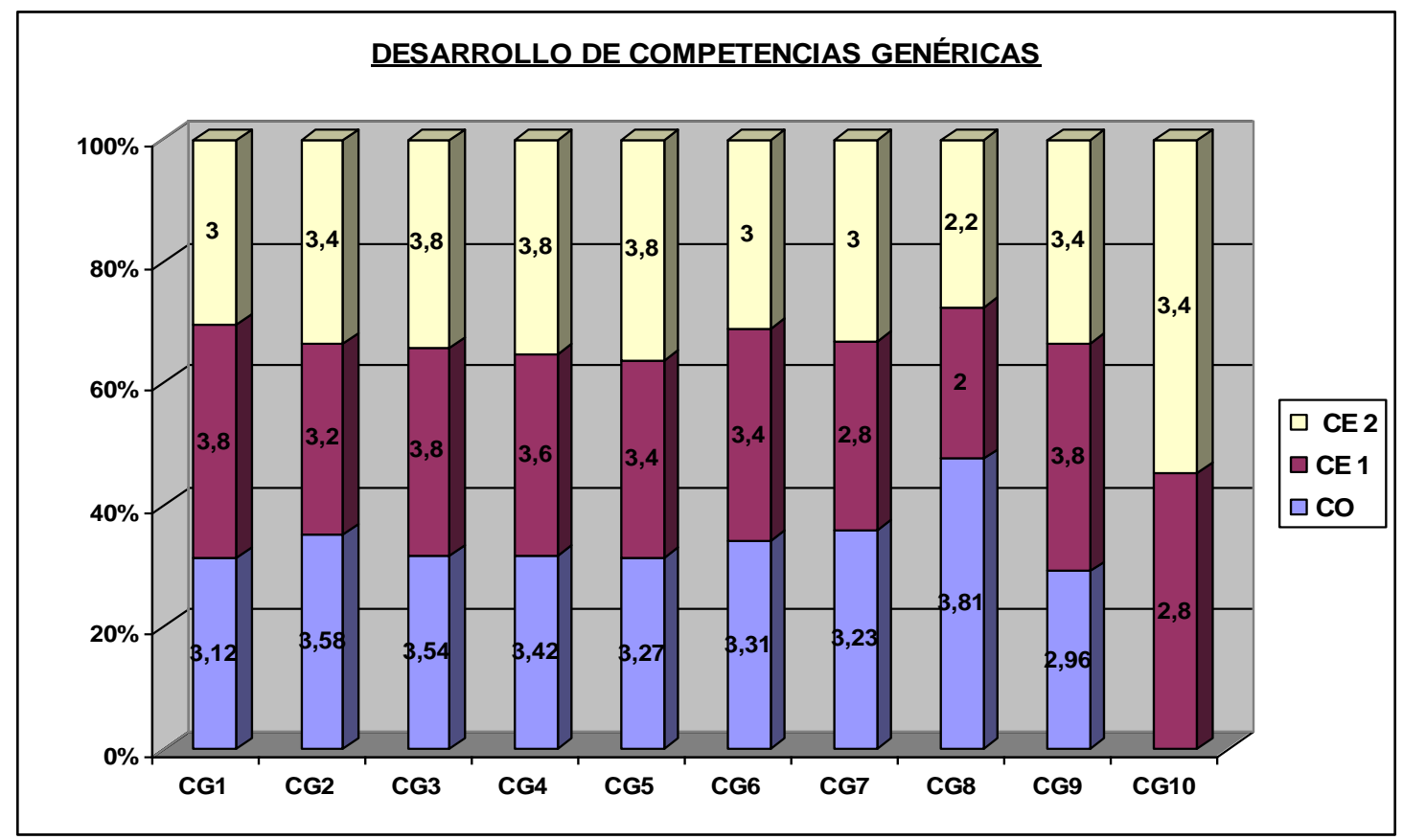

Diagrama 2. Desarrollo de competencias genéricas en los tres seminarios.

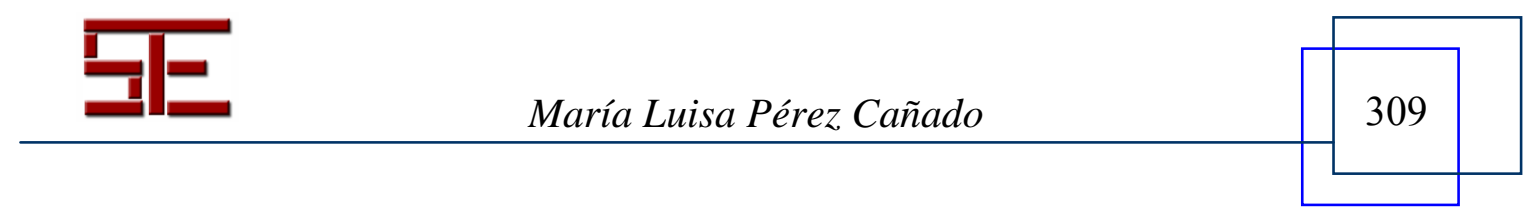




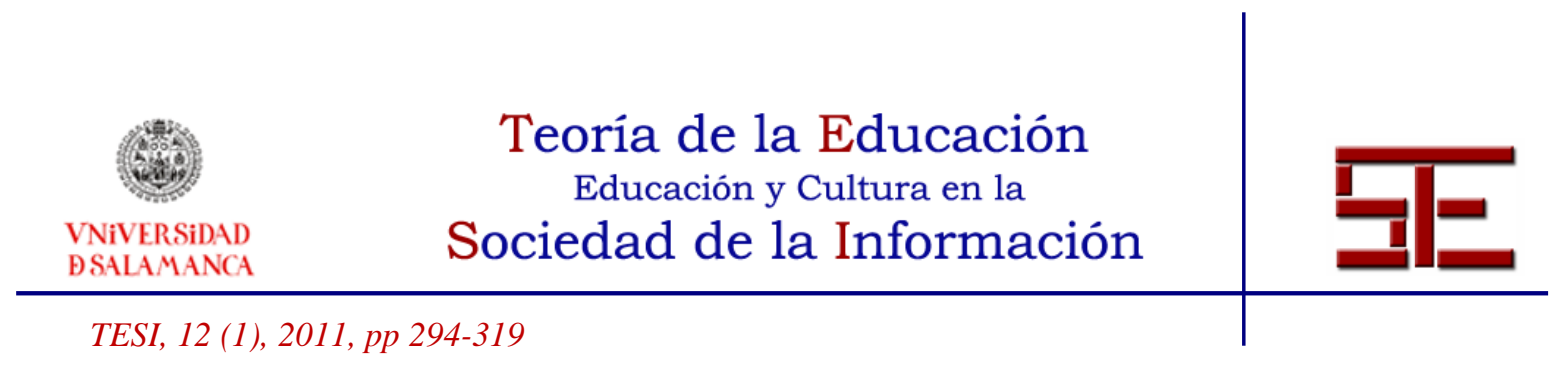

Destacamos que los seminarios sobre competencia escrita obtienen una puntuación más baja en trabajo en equipo, frente al de competencia oral, debido a que éste último ha potenciado la realización de un proyecto de trabajo cooperativo y tutorización por iguales (peer tutoring) para realizar exposiciones orales sobre temas de gramática inglesa. El compromiso ético prima más en el seminario sobre ensayos académicos al haberse centrado en gran medida en el tema del plagio y cómo resolverlo. Por último, hay que destacar que, interesantemente, en los seminarios sobre competencia escrita hay una mayor desviación típica a pesar de tener menos alumnos $\mathrm{y}$, en general, en estas variables hay más variabilidad de opiniones que en las relacionadas con la valoración de las sesiones, algo que se constata en los mínimos de 3, 2 e incluso 1 obtenidos en algunos apartados. Estos datos se pueden observar con detalle en las siguientes tablas:

\begin{tabular}{|c|c|c|c|c|c|c|c|c|c|}
\hline $\begin{array}{c}\text { EXPOSICIONES } \\
\text { ORALES }\end{array}$ & CG1 & CG2 & CG3 & CG4 & CG5 & CG6 & CG7 & CG8 & CG9 \\
\hline N & $\mathbf{3 1}$ & $\mathbf{3 1}$ & $\mathbf{3 1}$ & $\mathbf{3 1}$ & $\mathbf{3 1}$ & $\mathbf{3 1}$ & $\mathbf{3 1}$ & $\mathbf{3 1}$ & 31 \\
Media & $\mathbf{3 , 1 2}$ & $\mathbf{3 , 5 8}$ & $\mathbf{3 , 5 4}$ & $\mathbf{3 , 4 2}$ & $\mathbf{3 , 2 7}$ & $\mathbf{3 , 3 1}$ & $\mathbf{3 , 2 3}$ & $\mathbf{3 , 8 1}$ & 2,96 \\
Mediana & $\mathbf{3}$ & $\mathbf{4}$ & $\mathbf{4}$ & $\mathbf{3 , 5}$ & $\mathbf{3}$ & $\mathbf{3}$ & $\mathbf{3}$ & $\mathbf{4}$ & 3 \\
Moda & $\mathbf{3}$ & $\mathbf{4}$ & $\mathbf{4}$ & $\mathbf{4}$ & $\mathbf{3}$ & $\mathbf{3}$ & $\mathbf{3}$ & $\mathbf{4}$ & 3 \\
Desv. típ. & $\mathbf{0 , 5 2}$ & $\mathbf{0 , 5}$ & $\mathbf{0 , 5 8}$ & $\mathbf{0 , 6 4}$ & $\mathbf{0 , 5 3}$ & $\mathbf{0 , 4 7}$ & $\mathbf{0 , 5 9}$ & $\mathbf{0 , 4}$ & 0,77 \\
Mínimo & $\mathbf{2}$ & $\mathbf{3}$ & $\mathbf{2}$ & $\mathbf{2}$ & $\mathbf{2}$ & $\mathbf{3}$ & $\mathbf{2}$ & $\mathbf{3}$ & 1 \\
Máximo & 4 & 4 & 4 & 4 & 4 & 4 & 4 & 4 & 4 \\
\hline
\end{tabular}

Tabla 6. Competencias genéricas desarrolladas en el seminario sobre competencia oral.

\begin{tabular}{|c|c|c|c|c|c|c|c|c|c|c|}
\hline $\begin{array}{c}\text { ENSAYOS } \\
\text { LITERARIOS }\end{array}$ & CG1 & CG2 & CG3 & CG4 & CG5 & CG6 & CG7 & CG8 & CG9 & CG10 \\
\hline N & $\mathbf{5}$ & $\mathbf{5}$ & $\mathbf{5}$ & $\mathbf{5}$ & $\mathbf{5}$ & $\mathbf{5}$ & $\mathbf{5}$ & $\mathbf{5}$ & $\mathbf{5}$ & 5 \\
Media & $\mathbf{3 , 8}$ & $\mathbf{3 , 2}$ & $\mathbf{3 , 8}$ & $\mathbf{3 , 6}$ & $\mathbf{3 , 4}$ & $\mathbf{3 , 4}$ & $\mathbf{2 , 8}$ & $\mathbf{2}$ & $\mathbf{3 , 8}$ & 2,8 \\
Mediana & $\mathbf{4}$ & $\mathbf{3}$ & $\mathbf{4}$ & $\mathbf{4}$ & $\mathbf{3}$ & $\mathbf{4}$ & $\mathbf{2}$ & $\mathbf{2}$ & $\mathbf{4}$ & 4 \\
Moda & $\mathbf{4}$ & $\mathbf{3}$ & $\mathbf{4}$ & $\mathbf{4}$ & $\mathbf{3}$ & $\mathbf{4}$ & $\mathbf{2}$ & $\mathbf{1}$ & $\mathbf{4}$ & 4 \\
Desv. típ. & $\mathbf{0 , 4 5}$ & $\mathbf{0 , 8 4}$ & $\mathbf{0 , 4 5}$ & $\mathbf{0 , 5 5}$ & $\mathbf{0 , 5 5}$ & $\mathbf{0 , 8 9}$ & $\mathbf{1 , 1}$ & $\mathbf{1}$ & $\mathbf{0 , 4 5}$ & 1,64 \\
Mínimo & $\mathbf{3}$ & $\mathbf{2}$ & $\mathbf{3}$ & $\mathbf{3}$ & $\mathbf{3}$ & $\mathbf{2}$ & $\mathbf{2}$ & $\mathbf{1}$ & $\mathbf{3}$ & 1 \\
Máximo & 4 & 4 & 4 & 4 & 4 & 4 & 4 & 3 & 4 & 4 \\
\hline
\end{tabular}

Tabla 7. Competencias genéricas desarrolladas en el seminario sobre competencia escrita: ensayos literarios.

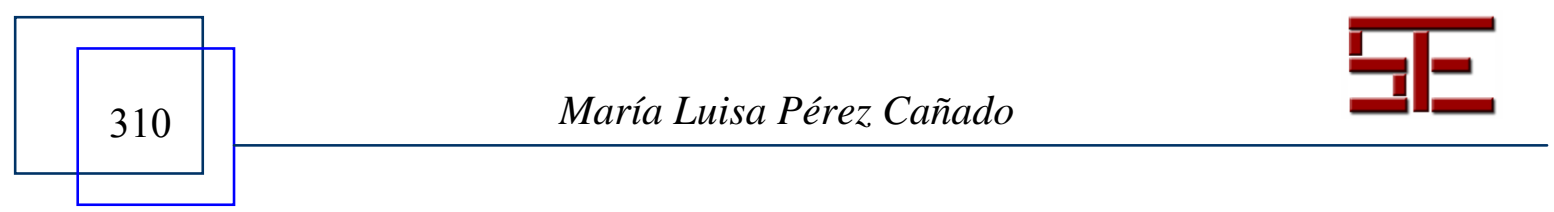




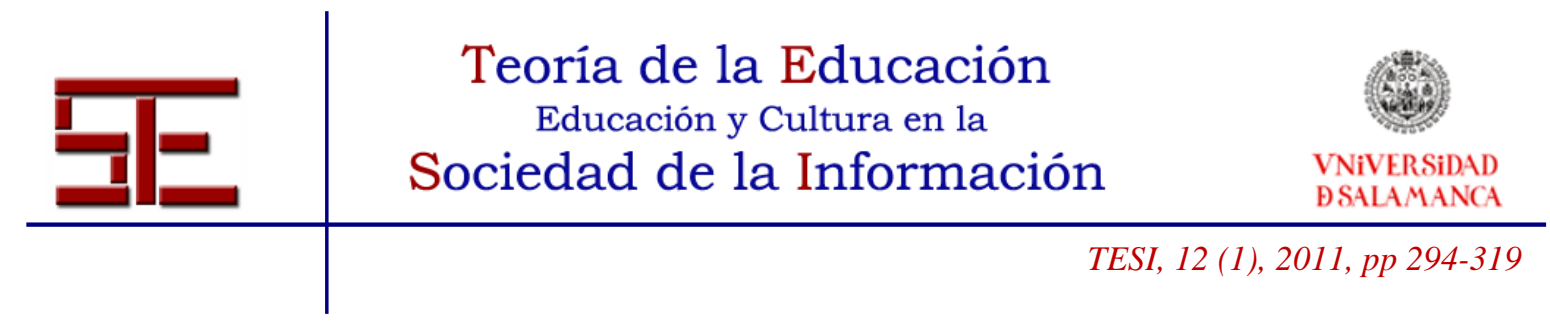

\begin{tabular}{|c|c|c|c|c|c|c|c|c|c|c|}
\hline $\begin{array}{c}\text { ENSAYOS } \\
\text { ACADÉMICOS }\end{array}$ & CG1 & CG2 & CG3 & CG4 & CG5 & CG6 & CG7 & CG8 & CG9 & CG10 \\
\hline N & $\mathbf{5}$ & $\mathbf{5}$ & $\mathbf{5}$ & $\mathbf{5}$ & $\mathbf{5}$ & $\mathbf{5}$ & $\mathbf{5}$ & $\mathbf{5}$ & $\mathbf{5}$ & 5 \\
Media & $\mathbf{3}$ & $\mathbf{3 , 4}$ & $\mathbf{3 , 8}$ & $\mathbf{3 , 8}$ & $\mathbf{3 , 8}$ & $\mathbf{3}$ & $\mathbf{3}$ & $\mathbf{2 , 2}$ & $\mathbf{3 , 4}$ & 3,4 \\
Mediana & $\mathbf{3}$ & $\mathbf{3}$ & $\mathbf{4}$ & $\mathbf{4}$ & $\mathbf{4}$ & $\mathbf{3}$ & $\mathbf{3}$ & $\mathbf{2}$ & $\mathbf{4}$ & 3 \\
Moda & $\mathbf{3}$ & $\mathbf{3}$ & $\mathbf{4}$ & $\mathbf{4}$ & $\mathbf{4}$ & $\mathbf{2}$ & $\mathbf{3}$ & $\mathbf{2}$ & $\mathbf{4}$ & 3 \\
Desv. típ. & $\mathbf{0 , 7 1}$ & $\mathbf{0 , 5 5}$ & $\mathbf{0 , 4 5}$ & $\mathbf{0 , 4 5}$ & $\mathbf{0 , 4 5}$ & $\mathbf{1}$ & $\mathbf{0 , 7 1}$ & $\mathbf{0 , 8 4}$ & $\mathbf{0 , 8 9}$ & 0,55 \\
Mínimo & $\mathbf{2}$ & $\mathbf{3}$ & $\mathbf{3}$ & $\mathbf{3}$ & $\mathbf{3}$ & $\mathbf{2}$ & $\mathbf{2}$ & $\mathbf{1}$ & $\mathbf{2}$ & 3 \\
Máximo & 4 & 4 & 4 & 4 & 4 & 4 & 4 & 3 & 4 & 4 \\
\hline
\end{tabular}

Tabla 8. Competencias genéricas desarrolladas en el seminario sobre competencia escrita: ensayos académicos.

\section{Clave:}

CG1: Capacidad de análisis y síntesis.

CG2: Capacidad de aplicar los conocimientos en la práctica.

CG3: Conocimientos generales básicos sobre el área de estudio.

CG4: Capacidad de aprender.

CG5: Capacidad crítica y autocrítica.

CG6: Capacidad para generar nuevas ideas (creatividad).

CG7: Toma de decisiones.

CG8: Trabajo en equipo.

CG9: Habilidad para trabajar de forma autónoma.

CG10: Compromiso ético.

\subsection{3. - Competencias específicas}

Si comparamos la valoración otorgada a las competencias específicas en los tres seminarios, constatamos que es, una vez más, altamente positiva en prácticamente todos los casos (véase Diagrama 3). Destacan especialmente las altas puntuaciones que reciben las competencias que precisamente más nos interesan: dominio instrumental de la lengua inglesa, capacidad de comunicación oral y escrita en lengua inglesa y capacidad para comunicar y enseñar conocimientos adquiridos.

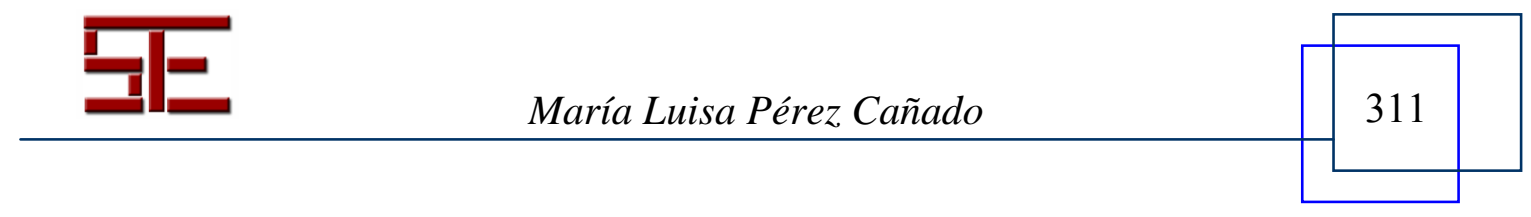



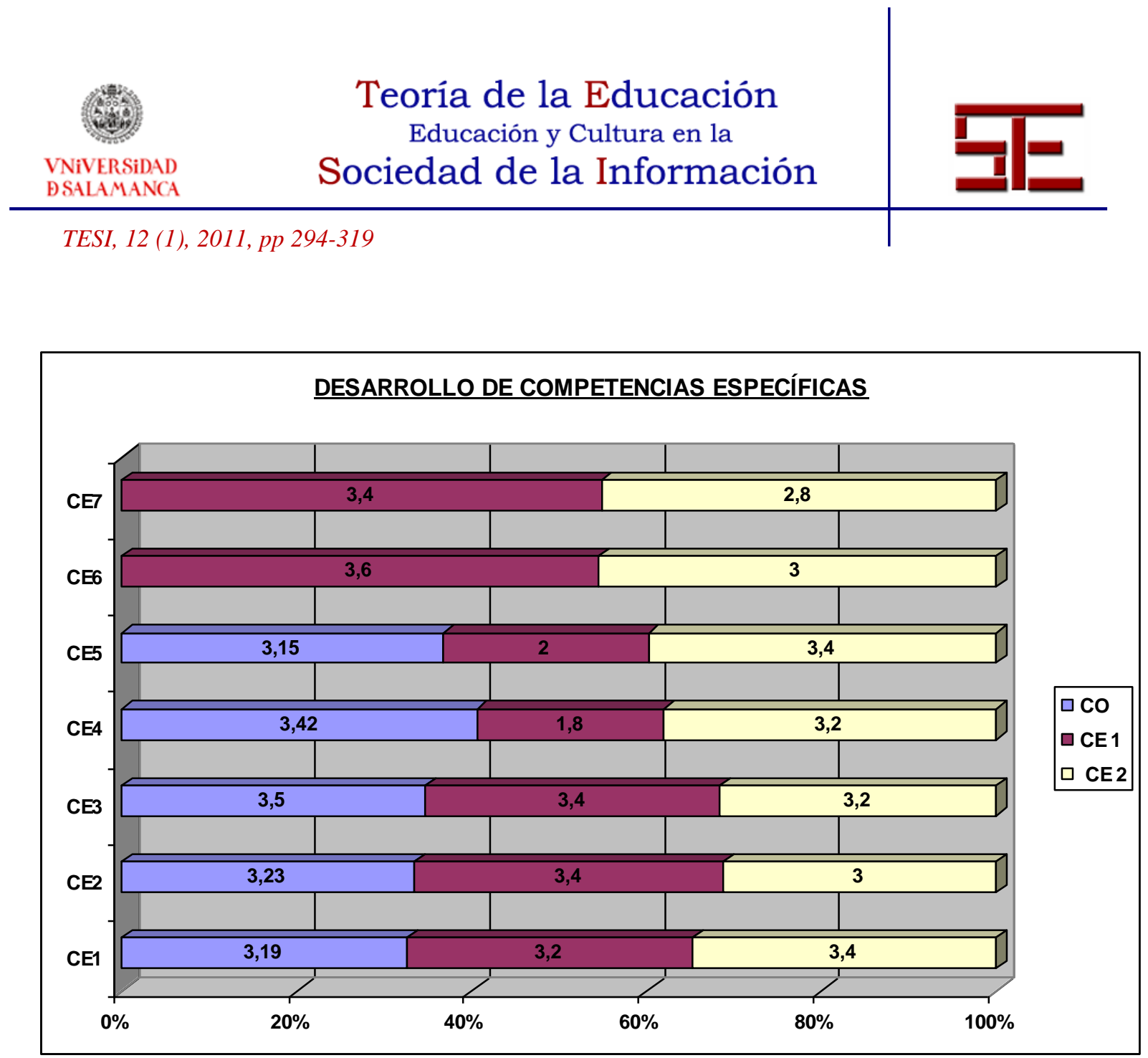

Diagrama 3. Desarrollo de competencias específicas en los tres seminarios.

Sin embargo, si analizamos los resultados por seminarios, observamos diferencias. En el caso de la comunicación oral (primer ciclo), la media supera siempre el valor 3 (véase Tabla 9). Así, aunque en algunas ocasiones se han producido respuestas tipo 1 o 2 , no son significativas. Los estadísticos mediana y moda confirman los resultados de la media. Podemos concluir, pues, que los resultados son altamente positivos.

\begin{tabular}{|c|c|c|c|c|c|}
\hline $\begin{array}{c}\text { EXPOSICIONES } \\
\text { ORALES }\end{array}$ & CE1 & CE2 & CE3 & CE4 & CE5 \\
\hline N & $\mathbf{3 1}$ & $\mathbf{3 1}$ & $\mathbf{3 1}$ & $\mathbf{3 1}$ & 31 \\
Media & $\mathbf{3 , 1 9}$ & $\mathbf{3 , 2 3}$ & $\mathbf{3 , 5}$ & $\mathbf{3 , 4 2}$ & 3,15 \\
Mediana & $\mathbf{3}$ & $\mathbf{3}$ & $\mathbf{4}$ & $\mathbf{3}$ & 3 \\
Moda & $\mathbf{3}$ & $\mathbf{3}$ & $\mathbf{4}$ & $\mathbf{3}$ & 3 \\
Desv. típ. & $\mathbf{0 , 6 9}$ & $\mathbf{0 , 5 9}$ & $\mathbf{0 , 5 8}$ & $\mathbf{0 , 5 8}$ & 0,61 \\
Mínimo & $\mathbf{2}$ & $\mathbf{2}$ & $\mathbf{2}$ & $\mathbf{2}$ & 2 \\
Máximo & 4 & 4 & 4 & 4 & 4 \\
\hline
\end{tabular}

Tabla 9. Competencias específicas desarrolladas en el seminario sobre competencia oral.

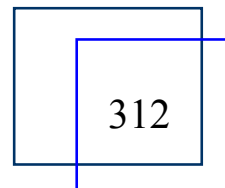




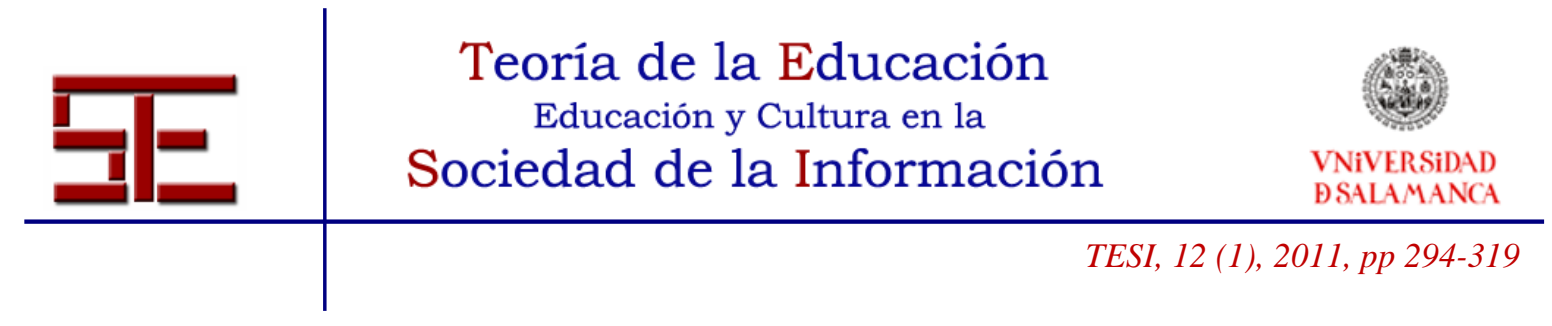

Si consideramos el seminario sobre ensayos académicos (véase Tabla 10), la variabilidad que se produce es alta, como se desprende de la desviación típica, por lo que quizás la mediana y la moda sean estadísticos más representativos que la media, siendo más altos que esta última.

\begin{tabular}{|c|c|c|c|c|c|c|c|}
\hline $\begin{array}{c}\text { ENSAYOS } \\
\text { LITERARIOS }\end{array}$ & CE1 & CE2 & CE3 & CE4 & CE5 & CE 6 & CE7 \\
\hline N & $\mathbf{5}$ & $\mathbf{5}$ & $\mathbf{5}$ & $\mathbf{5}$ & $\mathbf{5}$ & $\mathbf{5}$ & 5 \\
Media & $\mathbf{3 , 2}$ & $\mathbf{3 , 4}$ & $\mathbf{3 , 4}$ & $\mathbf{1 , 8}$ & $\mathbf{2}$ & $\mathbf{3 , 6}$ & 3,4 \\
Mediana & $\mathbf{4}$ & $\mathbf{3}$ & $\mathbf{4}$ & $\mathbf{2}$ & $\mathbf{2}$ & $\mathbf{4}$ & 4 \\
Moda & $\mathbf{4}$ & $\mathbf{3}$ & $\mathbf{4}$ & $\mathbf{1}$ & $\mathbf{1}$ & $\mathbf{4}$ & 4 \\
Desv. típ. & $\mathbf{1 , 3}$ & $\mathbf{0 , 5 5}$ & $\mathbf{0 , 8 9}$ & $\mathbf{0 , 8 4}$ & $\mathbf{1}$ & $\mathbf{0 , 5 5}$ & 0,89 \\
Mínimo & $\mathbf{1}$ & $\mathbf{3}$ & $\mathbf{2}$ & $\mathbf{1}$ & $\mathbf{1}$ & $\mathbf{3}$ & 2 \\
Máximo & 4 & 4 & 4 & 3 & 3 & 4 & 4 \\
\hline
\end{tabular}

Tabla 10. Competencias específicas desarrolladas en el seminario sobre competencia escrita: ensayos literarios.

Por último, en la sesión sobre ensayos académicos y plagio, si bien sigue existiendo alta variabilidad, se da en menor medida que en el otro seminario sobre competencia escrita. El valor modal continúa siendo más alto, en general, que la media (véase Tabla 11).

\begin{tabular}{|c|c|c|c|c|c|c|c|}
\hline ENSAYOS & CE1 & CE2 & CE3 & CE4 & CE5 & CE 6 & CE7 \\
ACAD'RMICOS & $\mathbf{5}$ & $\mathbf{5}$ & $\mathbf{5}$ & $\mathbf{5}$ & $\mathbf{5}$ & $\mathbf{5}$ & 5 \\
Media & $\mathbf{3 , 4}$ & $\mathbf{3}$ & $\mathbf{3 , 2}$ & $\mathbf{3 , 2}$ & $\mathbf{3 , 4}$ & $\mathbf{3}$ & 2,8 \\
Mediana & $\mathbf{4}$ & $\mathbf{3}$ & $\mathbf{3}$ & $\mathbf{3}$ & $\mathbf{4}$ & $\mathbf{4}$ & 3 \\
Moda & $\mathbf{4}$ & $\mathbf{2}$ & $\mathbf{3}$ & $\mathbf{3}$ & $\mathbf{4}$ & $\mathbf{4}$ & 4 \\
Desv. típ. & $\mathbf{0 , 8 9}$ & $\mathbf{1}$ & $\mathbf{0 , 8 4}$ & $\mathbf{0 , 8 4}$ & $\mathbf{0 , 8 9}$ & $\mathbf{1 , 4 1}$ & 1,3 \\
Mínimo & $\mathbf{2}$ & $\mathbf{2}$ & $\mathbf{2}$ & $\mathbf{2}$ & $\mathbf{2}$ & $\mathbf{1}$ & 1 \\
Máximo & 4 & 4 & 4 & 4 & 4 & 4 & 4 \\
\hline
\end{tabular}

Tabla 11. Competencias específicas desarrolladas en el seminario sobre competencia escrita: ensayos académicos.

\section{Clave:}

CE1: Dominio instrumental de la lengua inglesa.

CE2: Capacidad de comunicación oral y escrita en lengua inglesa.

CE3: Capacidad para comunicar y enseñar los conocimientos adquiridos.

CE4: Capacidad de comunicación y perlocutiva.

CE5: Capacidad de análisis y síntesis de documentación compleja.

CE6: Conocimiento de retórica estilística.

CE7: Conocimiento de crítica textual y edición de textos.

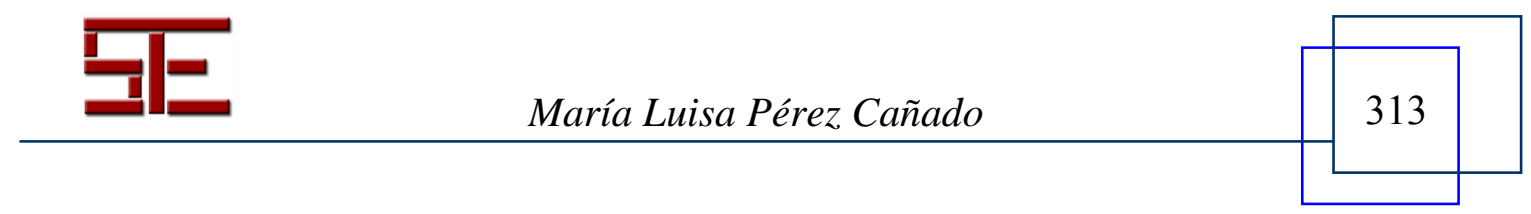




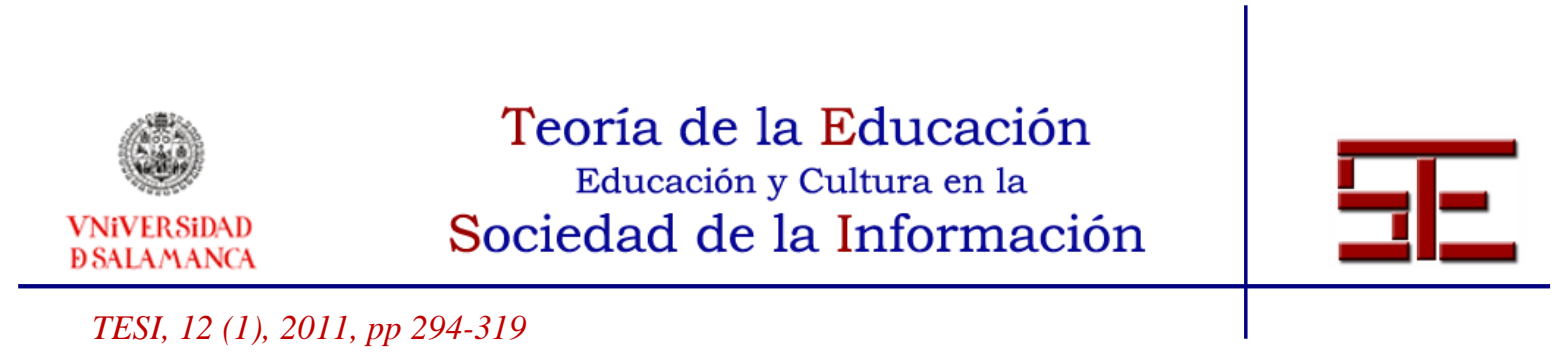

\section{4. - DISCUSIÓN}

Nuestros resultados nos permiten, por tanto, confirmar las hipótesis que nos planteábamos al inicio del estudio.

En primer lugar, los tres seminarios ECTS para el desarrollo de la competencia oral y escrita se han considerado adecuados y útiles. El alumnado participante ha valorado muy positivamente los temas sobre los que han versado, sus objetivos, contenidos, materiales, impartición y evaluación, algo que ha quedado patente tanto en las preguntas cerradas como en las abiertas.

También se ha constatado que los seminarios han proporcionado al alumnado las herramientas idóneas para afrontar con éxito la utilización de la comunicación oral y escrita en inglés, ya que este último ítem ha recibido una valoración entre 3,73 y 4 en una escala Likert de 4 puntos, y una moda y mediana de 4 en los tres casos.

Por último, se ha confirmado el éxito de los seminarios planteados para desarrollar competencias genéricas y específicas identificadas como claveS para el filólogo inglés. En efecto, el alumnado es altamente consciente del desarrollo de competencias genéricas relacionadas con capacidades tan esenciales para el EEES como la capacidad de análisis y síntesis, el aprendizaje a lo largo de toda la vida, la creatividad y toma de decisiones, el trabajo autónomo y en equipo, la capacidad crítica o el compromiso ético. Las competencias específicas son también muy positivamente valoradas, especialmente las que tienen que ver con el dominio instrumental de la lengua inglesa, la capacidad de comunicación oral y escrita en lengua inglesa o la capacidad de comunicar y enseñar los conocimientos adquiridos. Se confirma, pues, el éxito de la iniciativa en todos los elementos considerados.

\section{5. - CONCLUSIÓN}

En el presente artículo, hemos descrito una experiencia novedosa en el desarrollo de competencias genéricas y específicas y en el fomento de la interdisciplinariedad y la transversalidad. Se trata de un sistema de seminarios ECTS -que también conectan con el Plan de Acción Tutorial- que se han desarrollado en la Licenciatura en Filología Inglesa de la Universidad de Jaén durante el presente curso académico (2008-2009).

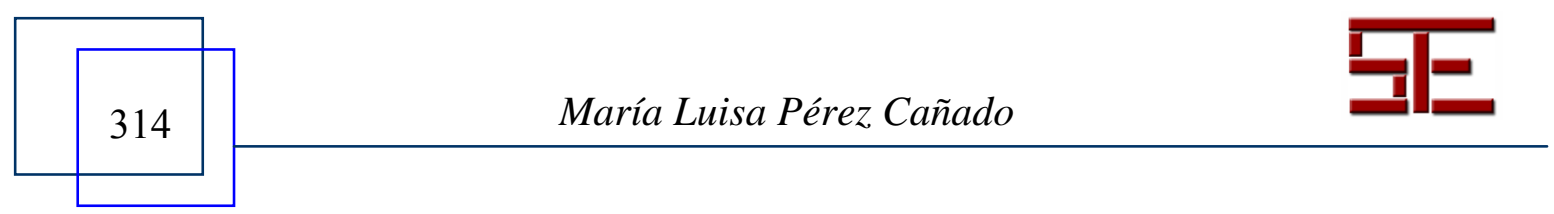




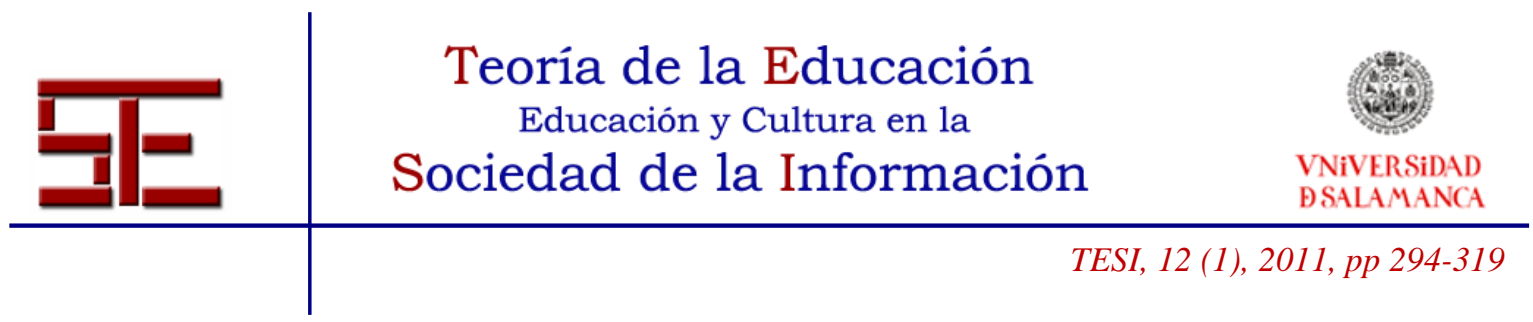

Con ellos, hemos pretendido fomentar la coordinación entre el profesorado de dicha titulación, superar el individualismo que tradicionalmente caracteriza a las actuaciones docentes en educación terciaria y realizar la transición desde la cultura tradicional de la enseñanza memorista y transmisiva hacia la cultura del aprendizaje crítico y significativo basado en competencias y no meramente contenidos.

Tras justificar la necesidad de una experiencia de este tipo y describir su desarrollo, contenidos y evaluación en detalle, hemos expuesto el estudio descriptivo que la ha acompañado. Dicha investigación nos ha permitido concluir que el sistema de seminarios ECTS ha resultado altamente beneficioso para el alumnado participante. Éste ha sido consciente de la existencia, desarrollo y utilidad de una serie de competencias genéricas y específicas relacionadas con la comunicación oral y escrita que todo graduado universitario ha de dominar. Consideramos que, en nuestro contexto, estos seminarios ECTS han constituido un importante primer paso en la integración, enseñanza y evaluación de competencias en el currículo, sin duda algo imprescindible para que nuestro alumnado pueda afrontar con éxito su vida personal, social y profesional en una sociedad incierta, compleja y cambiante como la actual.

\section{6. - REFERENCIAS}

Martín Ortega, M. E. (2008, diciembre). El papel de las concepciones de los docentes en los procesos de innovación. Ponencia presentada en las II Jornadas Internacionales UPM sobre Innovación Educativa y Convergencia Europea 2008. Universidad Politécnica de Madrid.

Michavila Pitarch, F. (2007, octubre). Conferencia Plenaria. II Jornadas de Trabajo sobre Experiencias Piloto en las Universidades Andaluzas. Universidad de Granada.

Organization for Economic Co-operation and Development (2003). Definition and Selection of Competencies: Theoretical and Conceptual Foundations (DeSeCo). Summary of the Final Report. Extraído el 9 de julio, 2010, de http://www.portalstat.admin.ch/deseco/deseco_final report_summary.pdf.

Pérez Cañado, M. L. (2010). English language teaching in the European Higher Education Area: from policy to practice. International Journal of Innovation in Language Learning and Teaching, 4, 1, 53-69.

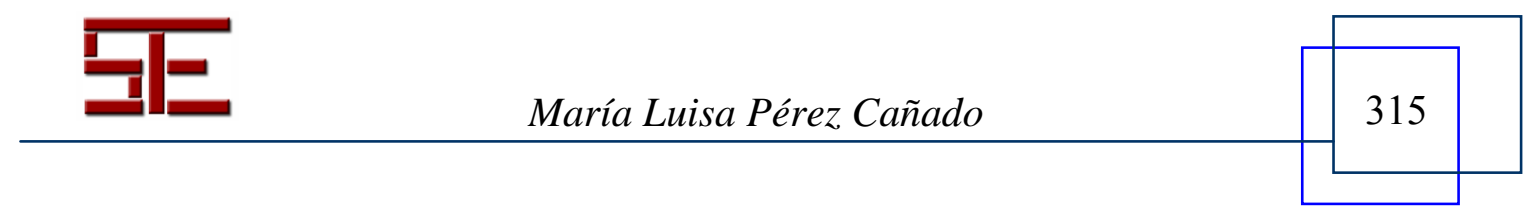




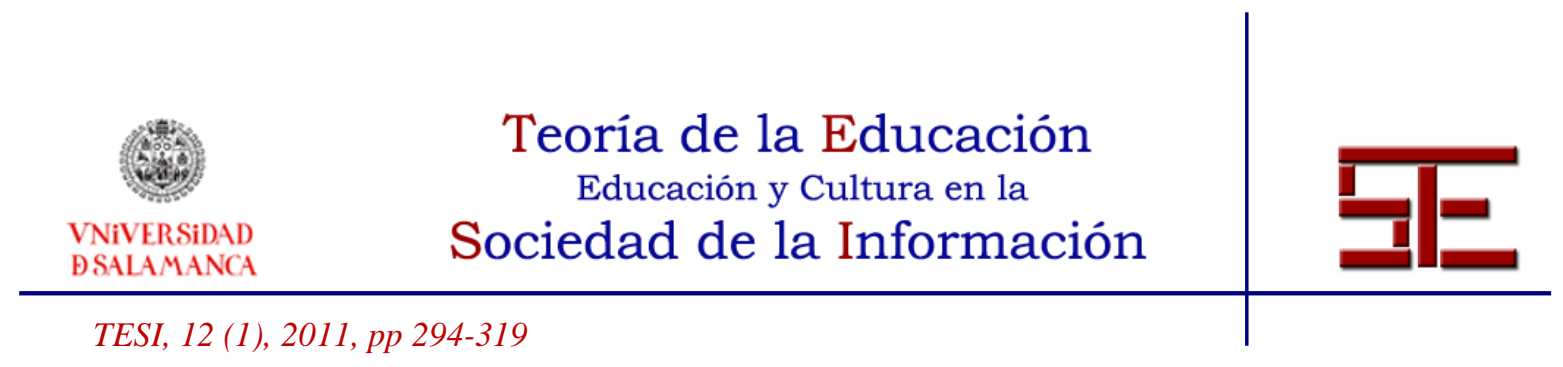

Pérez Cañado, M. L. \& Casas Pedrosa, A. V. (2010). La aplicación del crédito europeo a la titulación de Filología Inglesa en la Universidad de Jaén: análisis de debilidades y fortalezas. Revista Lenguaje y Textos, 31, 61-77.

Pérez Gómez, A., Soto Gómez, E., Sola Fernández, M. \& Serván Núñez, M. J. (2009a). Aprender en la universidad. El sentido del cambio en el EEES. Madrid: Ediciones Akal, S.A.

- (2009b). Los títulos universitarios y las competencias fundamentales: los tres ciclos. Madrid: Ediciones Akal, S.A.

- . (2009c). Aprender cómo aprender. Autonomía y responsabilidad: el aprendizaje de los estudiantes. Madrid: Ediciones Akal, S.A.

- (2009d). Orientar el desarrollo de competencias y enseñar cómo aprender. La tarea del docente. Madrid: Ediciones Akal, S.A.

- (2009e). Contextos y recursos para el aprendizaje relevante en la universidad. Madrid: Ediciones Akal, S.A.

Pérez González, J. (2009). Competencies in language teaching: from their conceptualisation to their concretion in the curriculum. En M. L. Pérez Cañado (Ed.), English language teaching in the European Credit Transfer System: from theory to practice (pp. 93-108). Franfurkt am Main: Peter Lang.

Poblete Ruiz, M. (2008, diciembre). Cuestiones clave en torno a la formación basada en competencias. Ponencia presentada en las II Jornadas Internacionales UPM sobre Innovación Educativa y Convergencia Europea 2008. Universidad Politécnica de Madrid.

Zabalza Beraza, M. A. (2004). Guía para la Planificación Didáctica de la Docencia Universitaria en el Marco del EEES. Santiago de Compostela: Universidad de Santiago de Compostela.

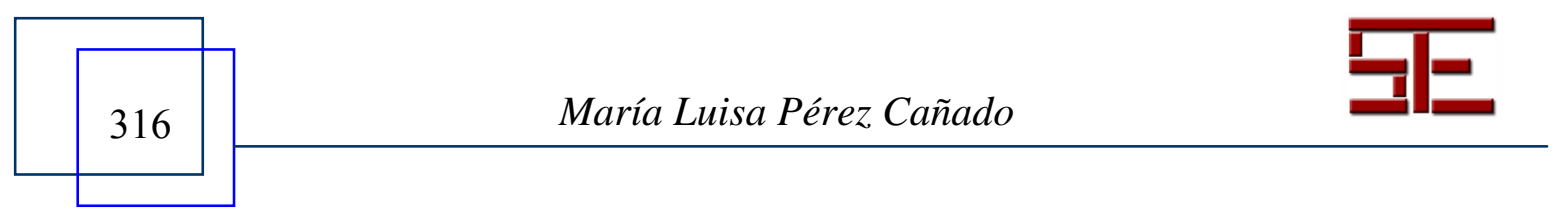




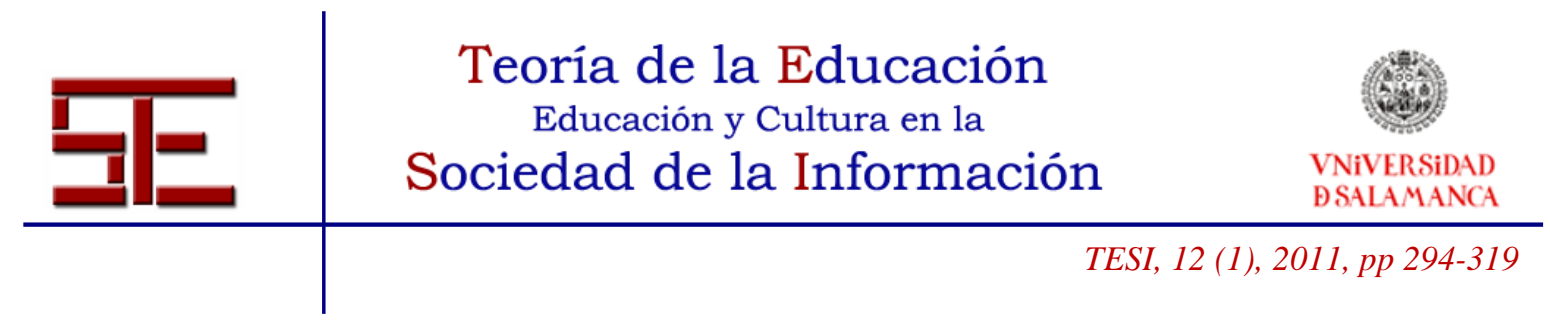

APÉNDICE I

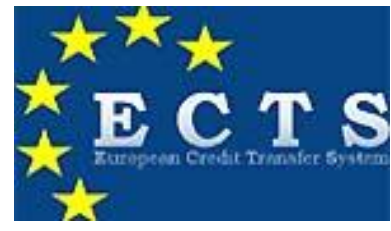

ECTS Cross-curricular Seminars

How to write an essay-like commentary: practical quidelines and basic terminology for literary analysis

Session by Prof. Juan Ráez Padilla and Prof. Yolanda Caballero Aceituno University of Jaén

December $10^{\text {th }}, 2008$

\begin{tabular}{|l|l|}
\hline NAME: \\
\hline OBJECTIVES: \\
\hline SUMMARY OF CONTENT: \\
\hline MAIN CONCLUSIONS: \\
\hline COMMENTS AND OPINION: \\
\hline \\
\hline
\end{tabular}




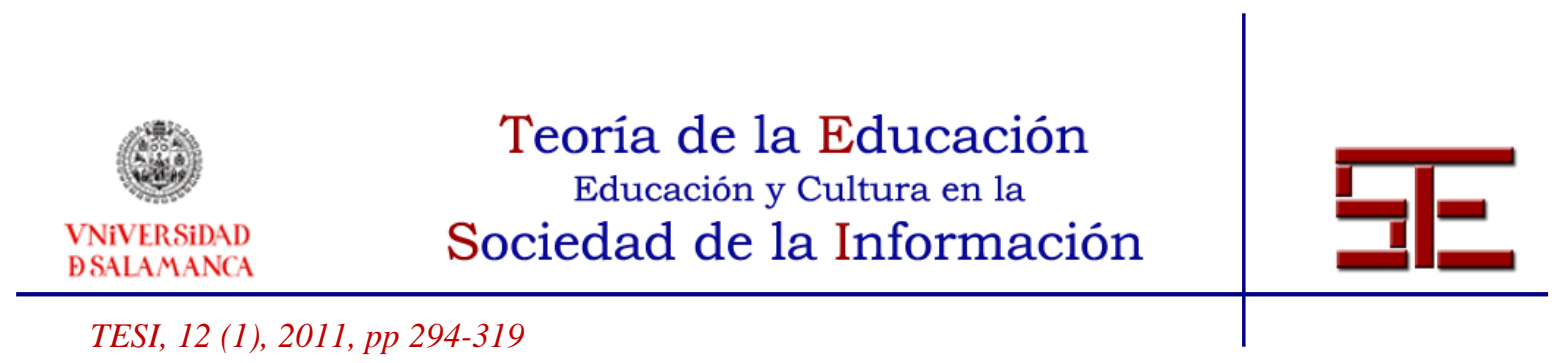

\section{APÉNDICE II}

SEMINARIOS TRANSVERSALES ECTS

"How to write an essay-like commentary: practical guidelines and basic terminology for literary analysis"

December $10^{\text {th }}, 2008$

\section{CURSO:}

Por favor, expresa tu opinión sobre los siguientes aspectos rodeando el número que mejor refleje lo que piensas:

$1=$ Nada (no estás de acuerdo) $\quad 2=$ Poco (estás poco de acuerdo) $\quad 3=$ Bastante (estás bastante de acuerdo) $\quad 4=$ Mucho (estás muy de acuerdo)

\begin{tabular}{|c|c|c|c|c|}
\hline Aspectos del seminario & & & & \\
\hline El tema del seminario ha sido útil. & 1 & 2 & 3 & 4 \\
\hline Los objetivos del seminario han estado claramente establecidos. & 1 & 2 & 3 & 4 \\
\hline Los contenidos del seminario han sido útiles. & 1 & 2 & 3 & 4 \\
\hline Los materiales del seminario se han preparado y presentado adecuadamente. & 1 & 2 & 3 & 4 \\
\hline La impartición del seminario ha sido adecuada. & 1 & 2 & 3 & 4 \\
\hline Los materiales para evaluar el seminario han sido adecuados. & 1 & 2 & 3 & 4 \\
\hline $\begin{array}{l}\text { Este seminario me ha proporcionado las herramientas idóneas para realizar comentarios literarios con } \\
\text { confianza. }\end{array}$ & 1 & 2 & 3 & 4 \\
\hline
\end{tabular}

Señala, por favor, cuáles de las siguientes competencias genéricas y específicas consideras que se han trabajado en este seminario, rodeando el número que mejor refleje lo que opinas (1=Nada; 2=Poco; 3=Bastante; 4=Mucho).

\begin{tabular}{|c|c|c|c|c|}
\hline Competencias genéricas desarrolladas & & & & \\
\hline Capacidad de análisis y síntesis & 1 & 2 & 3 & 4 \\
\hline Capacidad de aplicar los conocimientos en la práctica & 1 & 2 & 3 & 4 \\
\hline Conocimientos generales básicos sobre el área de estudio & 1 & 2 & 3 & 4 \\
\hline Capacidad de aprender & 1 & 2 & 3 & 4 \\
\hline Capacidad crítica y autocrítica & 1 & 2 & 3 & 4 \\
\hline Capacidad para generar nuevas ideas (creatividad) & 1 & 2 & 3 & 4 \\
\hline Toma de decisiones & 1 & 2 & 3 & 4 \\
\hline Trabajo en equipo & 1 & 2 & 3 & 4 \\
\hline Apreciación de la diversidad y multiculturalidad & 1 & 2 & 3 & 4 \\
\hline Conocimiento de culturas y costumbres de otros países & 1 & 2 & 3 & 4 \\
\hline Habilidad para trabajar de forma autónoma & 1 & 2 & 3 & 4 \\
\hline
\end{tabular}

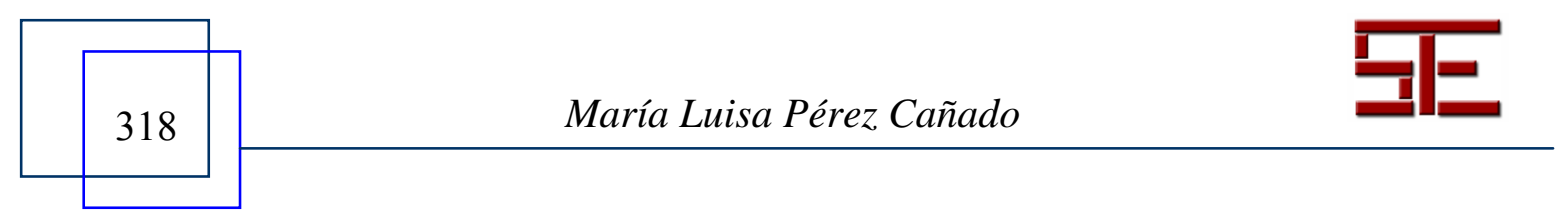




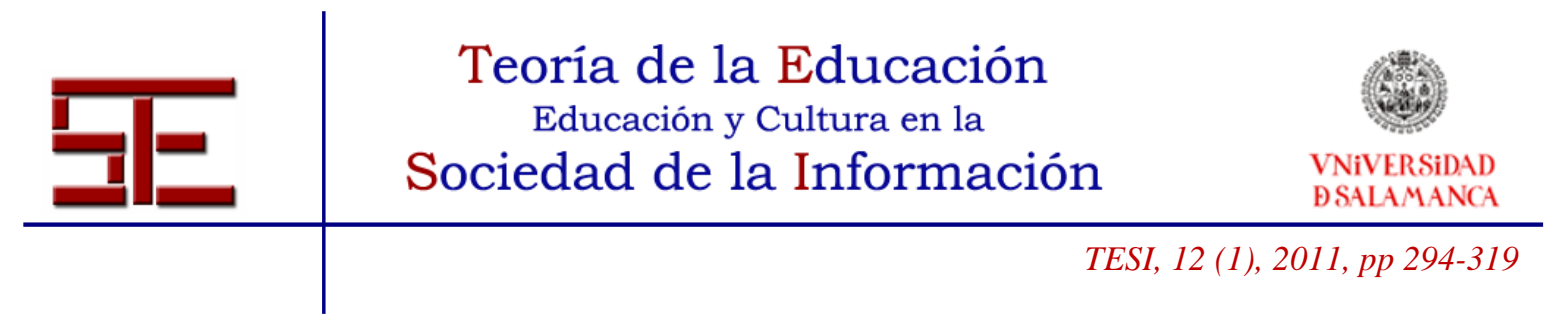

\begin{tabular}{|c|c|c|c|c|}
\hline Competencias específicas desarrolladas & & & & \\
\hline Dominio instrumental de la lengua inglesa & 1 & 2 & 3 & 4 \\
\hline Capacidad de comunicación oral y escrita en lengua inglesa & 1 & 2 & 3 & 4 \\
\hline Capacidad para comunicar y enseñar los conocimientos adquiridos & 1 & 2 & 3 & 4 \\
\hline Capacidad para localizar, manejar y sintetizar información bibliográfica & 1 & 2 & 3 & 4 \\
\hline Capacidad para realizar labores de asesoramiento y corrección lingüística & 1 & 2 & 3 & 4 \\
\hline Capacidad de comunicación y perlocutiva & 1 & 2 & 3 & 4 \\
\hline Capacidad de análisis y síntesis de documentación compleja & 1 & 2 & 3 & 4 \\
\hline
\end{tabular}

Comenta cualquier otro aspecto digno de mención acerca del seminario ECTS:

Para citar el presente artículo puede utilizar la siguiente referencia:

Pérez Cañado, M. L. (2011). El desarrollo de competencias comunicativas a través de seminarios transversales ects: una experiencia en la Universidad de Jaén, en Hernández Serrano, M. J. y Fuentes Agustí, M. (Coords.) La red como recurso de información en educación. Revista Teoría de la Educación: Educación y Cultura en la Sociedad de la Información. Vol. 12, n 1 . Universidad de Salamanca, pp. 294-319 [Fecha de consulta: $\mathrm{dd} / \mathrm{mm} / \mathrm{aaaa}]$.

http://campus.usal.es/ revistas_trabajo/index.php/revistatesi/article/view/7834/7860 